\title{
ON HIGHER-ORDER PROBABILISTIC SUBRECURSION
}

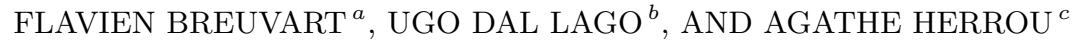 \\ ${ }^{a}$ LIPN, Université Paris Nord, France \\ e-mail address: flavien.breuvart@lipn.univ-paris13.fr \\ ${ }^{b}$ DISI, Università di Bologna, Italy \\ e-mail address: ugo.dallago@unibo.it \\ ${ }^{c}$ LIRIS, Université Claude Bernard 1, France \\ e-mail address: agathe.herrou@liris.cnrs.fr
}

\begin{abstract}
We study the expressive power of subrecursive probabilistic higher-order calculi. More specifically, we show that endowing a very expressive deterministic calculus like Gödel's $\mathbb{T}$ with various forms of probabilistic choice operators may result in calculi which are not equivalent as for the class of distributions they give rise to, although they all guarantee almost-sure termination. Along the way, we introduce a probabilistic variation of the classic reducibility technique, and we prove that the simplest form of probabilistic choice leaves the expressive power of $\mathbb{T}$ essentially unaltered. The paper ends with some observations about the functional expressive power: expectedly, all the considered calculi capture the functions which $\mathbb{T}$ itself represents, at least when standard notions of observations are considered.
\end{abstract}

\section{INTRODUCTION}

Probabilistic models are more and more pervasive in computer science and are among the most powerful modeling tools in many areas like computer vision [Pri12], machine learning [Pea88] and natural language processing [MS99]. Since the early times of computation theory [DLMSS56], the very concept of an algorithm has been itself generalized from a purely deterministic process to one in which certain elementary computation steps can have a probabilistic outcome. This has further stimulated research in computation and complexity theory [Gil74], but also in programming languages [SD78, Koz81].

Endowing programs with probabilistic primitives (e.g., an operator which models sampling from a distribution) poses a challenge to programming language semantics. Already for a minimal, imperative probabilistic programming language, giving a denotational semantics is nontrivial [Koz81]. When languages also have higher-order constructs, everything becomes even harder [JT98] to the point of disrupting much of the beautiful theory known in the deterministic case [Bar84]. This has stimulated research about the denotational semantics of higher-order probabilistic programming languages, with some surprising positive results coming out recently (e.g., [EPT14, CDL14, DLFVY17, HKSY17]).

Key words and phrases: Randomized Computation, Lambda Calculus, Higher-Order Recursion.

DOI:10.46298/LMCS-17(4:25)2021

(c) F. Breuvart, U. Dal Lago, and A. Herrou

(c) Creative Commons 
Not much is known about the expressive power of probabilistic higher-order calculi, as opposed to the extensive literature on deterministic such calculi (see, e.g. [Sta79, SU06]). What happens to the class of representable functions if one enriches, say, a deterministic $\lambda$-calculus $\mathbb{X}$ with certain probabilistic choice primitives? Are the expressive power and/or the good properties of $\mathbb{X}$ somehow preserved? These questions have been given answers in the case in which $\mathbb{X}$ is the pure, untyped, $\lambda$-calculus [DLZ12]: in that case, the calculus stays universal, mimicking what happens in Turing machines [San69]. But what if $\mathbb{X}$ is one of the many typed $\lambda$-calculi ensuring strong normalization for typed terms [GTL89]?

Let us do a step back, first: when should a higher-order probabilistic program be considered terminating in the first place? The question can be given a satisfactory answer being inspired by, e.g., recent work on probabilistic termination in imperative languages and term rewrite systems [MM05, BG05]: one could ask the probability of divergence to be 0 , called the almost sure termination property, or the stronger positive almost sure termination property, in which one requires the average number of evaluation steps to be finite. That termination is desirable property, even in a probabilistic setting, can be seen, e.g. in the field of languages like CHURCH and ANGLICAN, in which programs are often assumed to be almost surely terminating, e.g. when doing inference by $\mathrm{MH}$ algorithms [GMR $\left.{ }^{+} 08\right]$ : if they are not, inference is bound to fail miserably.

In this paper, we initiate a study on the expressive power of terminating higher-order calculi, in particular those obtained by endowing Gödel's $\mathbb{T}$ with various forms of probabilistic choice operators. In particular, three operators will be analyzed in this paper:

- A binary probabilistic operator $\oplus$ such that for every pair of terms $M, N$, the term $M \oplus N$ evaluates to either $M$ or $N$, each with probability $\frac{1}{2}$. This is a rather minimal option, which, however, guarantees universality if applied to the untyped $\lambda$-calculus [DLZ12] (and, more generally, to universal models of computation [San69]).

- A combinator $\mathrm{R}$, which evaluates to any natural number $n \geq 0$ with probability $\frac{1}{2^{n+1}}$. This is the natural generalization of $\oplus$ to sampling from the geometric distribution which has countable rather than finite support. This apparently harmless generalization (which is absolutely non-problematic in the context of a Turing-complete computational model) has dramatic consequences in a subrecursive scenario, as we will discover soon.

- A combinator $\mathrm{x}$ such that for every pair of values $V, W$, the term $\mathrm{x}\langle V, W\rangle$ evaluates to either $W$ or $V(\mathrm{x}\langle V, W\rangle)$, each with probability $\frac{1}{2}$. The operator $\mathrm{X}$ can be seen as a probabilistic variation on $\mathbb{P C F}$ 's fixpoint combinator. As such, $\mathrm{X}$ is potentially problematic for termination, giving rise to infinite reduction trees.

This way, various calculi can be obtained, e.g., $\mathbb{T}^{\oplus}$, namely a minimal extension of $\mathbb{T}$, or the full calculus $\mathbb{T}^{\oplus, R, X}$, in which the three operators are all available. In principle, the only obvious fact about the expressive power of the above mentioned operators is that both $R$ and $\mathrm{X}$ are at least as expressive as $\oplus$ : binary choice can be easily expressed by either $\mathrm{R}$ or $\mathrm{X}$. Less obvious, but still easy to prove, is the equivalence between $\mathrm{R}$ and $\mathrm{X}$ in presence of an operator for primitive recursion (see Section 2.4). But how about, say, $\mathbb{T}^{\oplus}$ vs. $\mathbb{T}^{R}$ ?

Traditionally, the expressive power of such languages are compared by looking at the set of functions $f: \mathbb{N} \rightarrow \mathbb{N}$ defined by typable programs $M:$ NAT $\rightarrow$ NAT. However, in a probabilistic setting, programs $M:$ NAT $\rightarrow$ NAT computes functions from natural numbers to distributions of natural numbers. In order to evaluate the impact of the underlying probabilistic choice operator(s), we thus need to fix a notion of observation. There are at least two relevant notions of observations, corresponding to two randomized programming paradigms, namely the so-called Las Vegas and Monte Carlo observations [MR95, AB09]. 
The main problem, then, consists in understanding how the obtained classes relate to each other, and to the class of $\mathbb{T}$-representable functions, which is well-known to comprise precisely the provably total functions of Peano's arithmetic [GTL89]. Along the way, however, we manage to understand how to compare the expressive power of probabilistic calculi per se. Summing up, this paper's main contributions are the following ones:

- We first take a look at the full calculus $\mathbb{T}^{\oplus, R, X}$, and prove that it enforces almost-sure termination, namely that the probability of termination of any typable term is 1 . This is done by appropriately adapting the well-known reducibility technique [GTL89] to a probabilistic operational semantics. We then observe that while $\mathbb{T}^{\oplus, R, \mathrm{X}}$ cannot be positively almost surely terminating, $\mathbb{T}^{\oplus}$ indeed is. This already shows that there must be a gap in expressive power. This is done in Section 2.

- In Section 3, we look more closely at the expressive power of $\mathbb{T}^{\oplus}$, proving that the mere presence of probabilistic choice does not add much to the expressive power of $\mathbb{T}$ : in a sense, probabilistic choice can be "lifted up" to the ambient deterministic calculus.

- We look at other fragments of $\mathbb{T}^{\oplus, \mathrm{R}, \mathrm{X}}$ and at their expressive power. More specifically, we will prove that (the equiexpressive) $\mathbb{T}^{\mathrm{R}}$ and $\mathbb{T}^{\mathrm{X}}$ represent precisely what $\mathbb{T}^{\oplus}$ can do at the limit, in a sense which will be made precise in Section 2. This part, which turns out to be the most challenging, is in Section 4.

- Section 5 is devoted to proving that both for Monte Carlo and for Las Vegas observations, the class of functions representable in $\mathbb{T}^{\mathrm{R}}$ coincides with the $\mathbb{T}$-representable ones, the only exception being classes obtained by observing the most likely outcome, which are much larger and of questionable interest.

\section{Probabilistic Choice Operators, Informally}

Any term of Gödel's $\mathbb{T}$ can be seen as a purely deterministic computational object whose dynamics is finitary, due to the well-known strong normalization theorem (see, e.g., [GTL89]). In particular, the non-determinism due to multiple redex occurrences is completely harmless because of confluence. Confluence is well-known not to hold in a probabilistic scenario [DLZ12], but in this paper we neglect this problem, and work with a fixed reduction strategy, namely weak call-by-value reduction (keeping in mind that all what we say here also holds when call-by-name is the underlying notion of reduction). Evaluation of a $\mathbb{T}$-term $M$ of type NAT can be seen as a finite sequence of terms ending in the normal form $\boldsymbol{n}$ of $M$ (see Figure 1a). More generally, the unique normal form of any $\mathbb{T}$ term $M$ will be denoted as $\llbracket M \rrbracket$. Noticeably, $\mathbb{T}$ is computationally very powerful. In particular, the $\mathbb{T}$-representable functions on $\mathbb{N}$ coincide with the functions which are provably total in Peano's arithmetic [GTL89].

As we already mentioned, the most natural way to enrich deterministic calculi and turn them into probabilistic ones consists in endowing their syntax with one or more probabilistic choice operators. Operationally, each of them models the process of sampling from a distribution and proceeding depending on the outcome. Of course, one has many options here as for which one(s) of the various operators to grab. The aim of this work is precisely the one of studying to which extent this choice have consequences on the overall expressive power of the underlying calculus.

Suppose, for example, that $\mathbb{T}$ is endowed with the binary probabilistic choice operator $\oplus$ described in the Introduction, whose evaluation corresponds to tossing a fair coin and choosing one of the two arguments accordingly. The presence of $\oplus$ has indeed an impact on the dynamics of the underlying calculus: the evaluation of any term $M$ is not deterministic 


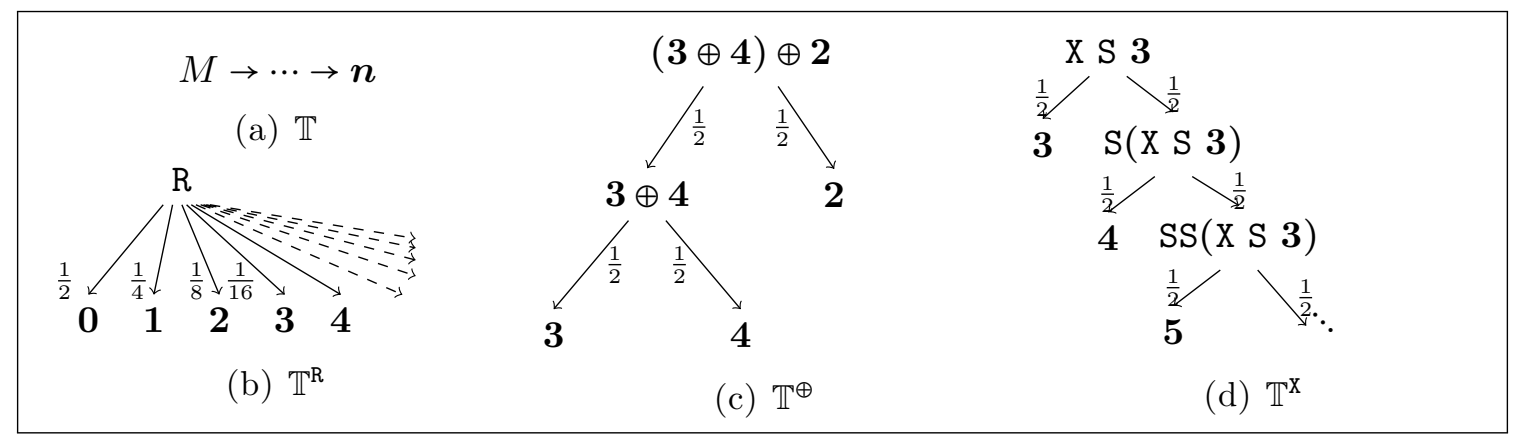

FiguRE 1. Reduction in Fragments of $\mathbb{T}^{\oplus, R, X}$

anymore, but can be modeled as a finitely branching tree (see, e.g. Figure 1c for such a tree when $M$ is $(\mathbf{3} \oplus \mathbf{4}) \oplus \mathbf{2})$. The fact that all branches of this tree have finite height (and the tree is thus finite) is intuitive, and a proof of it can be given by adapting the well-known reducibility proof of termination for $\mathbb{T}$. In this paper, we in fact prove much more, and establish that $\mathbb{T}^{\oplus}$ can be embedded into $\mathbb{T}$.

If $\oplus$ is replaced by $R$, the underlying tree is not finitely branching anymore, but, again, there is no infinitely long branch, since each of them can somehow be seen as a $\mathbb{T}$ computation (see Figure 1b for an example). What happens to the expressive power of the obtained calculus? Intuition tells us that the calculus should not be too expressive viz. $\mathbb{T}^{\oplus}$. If $\oplus$ is replaced by $\mathrm{X}$, on the other hand, the underlying tree is finitely branching, but its height can well be infinite (see Figure 1d). Actually, $X$ and $R$ are easily shown to be equiexpressive in presence of higher-order recursion, as we show in Section 2.4. On the other hand, for $\mathrm{R}$ and $\oplus$, no such result is possible. Nonetheless, $\mathbb{T}^{\mathrm{R}}$ can still be somehow encoded into $\mathbb{T}$, as we will detail in Section 4. From this embedding, we can show that neither Monte Carlo nor Las Vegas algorithms on $\mathbb{T}^{\oplus, X, R}$ add any expressive power to $\mathbb{T}$. This is done in Section 5 .

\section{The Full Calculus $\mathbb{T}^{\oplus, R, X}$}

All along this paper, we work with a calculus $\mathbb{T}^{\oplus, R, X}$ whose terms are the ones generated by the following grammar:

$$
M, N, L::=x|\lambda x . M| M N|\langle M, N\rangle| \pi_{1}\left|\pi_{2}\right| \operatorname{rec}|\mathbf{0}| \mathrm{S}|M \oplus N| \mathrm{R} \mid \mathrm{X} .
$$

Please observe the presence of the usual constructs from the untyped $\lambda$-calculus, but also of primitive recursion, constants for natural numbers, pairs, and the three choice operators we have described in the previous sections.

As usual, terms are taken modulo $\alpha$-equivalence. Terms in which no variable occurs free are, as usual, dubbed closed, and are collected in the set $\mathbb{T}_{C}^{\oplus, R, \mathrm{X}}$. A value is simply a closed term from the following grammar

$$
U, V::=\lambda x \cdot M|\langle U, V\rangle| \pi_{1}\left|\pi_{2}\right| \operatorname{rec}|\mathbf{0}| \mathrm{S}|\mathrm{S} V| \mathrm{X},
$$

and the set of all values is $\mathbb{T}_{V}^{\oplus, \mathrm{R}, \mathrm{X}}$. Closed terms that are not values are called reducible and their set is denoted $\mathbb{T}_{R}^{\oplus, \mathrm{R}, \mathrm{X}}$. A context is a term with a unique hole:

$$
C:=(\cdot)|\lambda x . C| C M|M C|\langle C, M\rangle|\langle M, C\rangle| C \oplus M \mid M \oplus C
$$




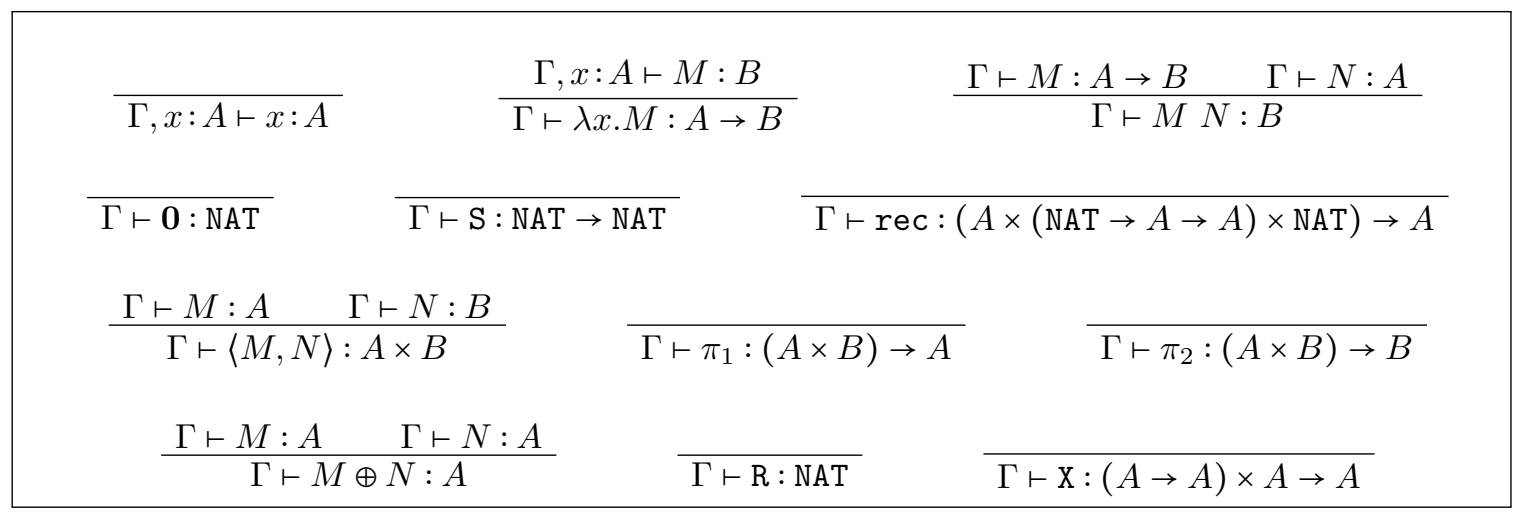

Figure 2. Typing Rules

We write $\mathbb{T}_{(\cdot)}^{\oplus, \mathrm{R}, \mathrm{X}}$ for the set of all such contexts. The expression $C(M)$ indicates the term obtained by substituting (.) with $M$ inside $C$.

Termination of Gödel's $\mathbb{T}$ is guaranteed by the presence of types, which we also need here. Types are expressions generated by the following grammar

$$
A, B::=\mathrm{NAT}|A \rightarrow B| A \times B .
$$

Environmental contexts are expressions of the form $\Gamma=x_{1}: A_{1}, \ldots, x_{n}: A_{n}$, while typing judgments are of the form $\Gamma \vdash M: A$. Typing rules are given in Figure 2. From now on, only typable terms will be considered. We denote as $\mathbb{T}_{C}^{\oplus, \mathrm{R}, \mathrm{X}}(A)$ the set of closed terms of type $A$, similarly for $\mathbb{T}_{V}^{\oplus, \mathrm{R}, \mathrm{X}}(A)$. Given $n \in \mathbb{N}$, we use the shortcut $\boldsymbol{n}$ for the corresponding value of type NAT: $\mathbf{0}$ is already part of the language of terms, while $\boldsymbol{n}+\mathbf{1}$ is simply $\mathrm{S} \boldsymbol{n}$. For simplicity of notations, we also write SS for $\lambda x . \mathrm{S}(\mathrm{S} x)$; similarly for SS ... S ( $n$ times).

2.1. Operational Semantics. While evaluating terms in a deterministic calculus ends up in a value, the same process leads to a distribution of values when applied to terms in a probabilistic calculus [DLZ12]. The reader should note that countable distributions are perfectly sufficient to give semantics to $\mathbb{T}^{\oplus, \mathrm{R}, \mathrm{X}}$, and that we will focus our attention on them here. Formalizing all this requires some care, but can be done following one of the many definitions from the literature (e.g., [DLZ12]).

Given a countable set $X$, a distribution $\mathcal{L}$ on $X$ is a function mapping elements of $X$ to elements of the interval $[0,1]$ :

$$
\mathcal{L}, \mathcal{M}, \mathcal{N} \in \mathfrak{D}(X)=\left\{f: X \rightarrow[0,1] \mid \sum_{x \in X} f(x) \leq 1\right\}
$$

Observe that we take distributions as functions summing to a real in $[0,1]$ rather than to 1 . This is an implicit way to handle computations with nonzero probability of divergence as distributions whose sum is strictly below 1 .

If $\mathcal{L}$ is a distribution on $X$ and $x \in X$, then $\mathcal{L}(x)$ is the real number which $\mathcal{L}$ put in correspondence to $x$. We will use the pointwise order $\leq$ on distributions, which turns them into an $\omega \mathbf{C P O}$. The support of a distribution $\mathcal{M} \in \mathfrak{D}(X)$, namely the subset of $X$ to which $\mathcal{M}$ assigns strictly positive probability, is indicated as $|\mathcal{M}|$. For any $\mathcal{M} \in \mathfrak{D}(X)$ and $Y \subseteq X$, $\mathcal{M}^{Y}$ is another distribution in $\mathfrak{D}(X)$ such that $\mathcal{M}^{Y}(x)=\mathcal{M}(x)$ if $x \in Y$ and $\mathcal{M}^{Y}(x)=0$ 
otherwise. Another useful operation on distributions is scalar multiplication: if $\mathcal{L} \in \mathfrak{D}(X)$ and $p \in[0,1]$ then $p \cdot \mathcal{L}$ is the distribution assigning to $x$ the probability $p \mathcal{L}(x)$.

We are especially interested in distributions over terms here. In particular, a distribution of type $A$ is simply an element of $\mathfrak{D}\left(\mathbb{T}_{C}^{\oplus, \mathrm{R}, \mathrm{X}}(A)\right)$ and, as such, it is a function from $\mathbb{T}_{C}^{\oplus, \mathrm{R}, \mathrm{X}}(A)$ to $[0,1]$ whose sum is itself less or equal to 1 . The set $\mathfrak{D}\left(\mathbb{T}_{V}^{\oplus, \mathrm{R}, \mathrm{X}}\right)$ is the set of of distribution over values and will thus be used to assign a meaning to close terms; it is ranged over by metavariables like $\mathcal{U}, \mathcal{V}, \mathcal{W}$. We use the following notation for Dirac's distributions over terms:

$$
\{M\}:=\left\{\begin{array}{l}
M \mapsto 1 \\
N \mapsto 0 \text { if } M \neq N
\end{array}\right\} .
$$

We define the reducible and value supports of a distribution $\mathcal{M}$ on terms as $|\mathcal{M}|_{R}:=|\mathcal{M}| \cap \mathbb{T}_{R}^{\oplus, \mathrm{R}, \mathrm{X}}$ and $|\mathcal{M}|_{V}:=|\mathcal{M}| \cap \mathbb{T}_{V}^{\oplus, R, \mathrm{X}}$, respectively. This way, notations like $\mathcal{M}^{R}$ and $\mathcal{M}^{V}$ have an obvious and natural meaning.

As syntactic sugar, we use the following convenient notation to manipulate distributions, i.e., for any family of distributions $\left(\mathcal{N}_{M}\right)_{M \in \mathbb{T}^{\oplus, \mathrm{R}, \mathrm{X}}}$ such that $\mathcal{N}_{M}$ is in $\mathfrak{D}\left(\mathbb{T}^{\oplus, \mathrm{R}, \mathrm{X}}\right)$ for every $M \in \mathbb{T}^{\oplus, \mathrm{R}, \mathrm{X}}$, the expression $\sum_{M \mapsto \mathcal{M}} \mathcal{N}_{M}$ stands for

$$
\sum_{M \mapsto \mathcal{M}} \mathcal{N}_{M}:=\sum_{M \in \mathbb{T}^{\oplus, \mathrm{R}, \mathrm{X}}} \mathcal{M}(M) \cdot \mathcal{N}_{M}
$$

By a slight abuse of notation, we may define $\mathcal{N}_{M}$ only for $M \in|\mathcal{M}|$, since the other elements of the family $\left(\mathcal{N}_{M}\right)_{M \in \mathbb{T}^{\oplus, R, \mathrm{x}}}$ do not anyway contribute to the sum. The sum notation as we use it here can be easily generalized, e.g., to families of real numbers $\left(p_{M}\right)_{M \in \mathbb{T}^{\oplus, \mathrm{R}, \mathrm{x}}}$ and to other kinds of distributions.

Example 2.1. Suppose that $\mathcal{M}=\left\{\boldsymbol{n} \mapsto \frac{1}{2^{n+1}} \mid n \in \mathbb{N}\right\} \in \mathfrak{D}\left(\mathbb{T}^{\oplus, \mathrm{R}, \mathrm{X}}(\mathrm{NAT})\right)$, which is the socalled exponential distribution over the natural numbers. Suppose, moreover, that for any $n, \mathcal{N}_{\boldsymbol{n}}=\left\{\begin{array}{cl}\boldsymbol{n}+\mathbf{1} & \mapsto \frac{1}{2} \\ \mathbf{0} & \mapsto \frac{1}{2}\end{array}\right\}$ is another distribution in $\mathfrak{D}\left(\mathbb{T}^{\oplus, \mathrm{R}, \mathrm{X}}(\mathrm{NAT})\right)$. Then

$$
\begin{aligned}
\sum_{M \mapsto \mathcal{M}} \mathcal{N}_{M} & =\sum_{n} \frac{1}{2^{n+1}} \cdot\left\{\begin{array}{cc}
\boldsymbol{n}+\mathbf{1} & \mapsto \frac{1}{2} \\
\mathbf{0} & \mapsto \frac{1}{2}
\end{array}\right\}=\left\{\begin{array}{ccc}
\boldsymbol{n}+\mathbf{1} & \mapsto & \frac{1}{2^{n+1}} \cdot \frac{1}{2} \\
\mathbf{0} & \mapsto & \sum_{m} \frac{1}{2^{m+1}}
\end{array}\right\} \\
& =\left\{\boldsymbol{n} \mapsto \frac{1}{2^{n+1}} \mid n \in \mathbb{N}\right\},
\end{aligned}
$$

which is exactly the same as $\mathcal{M}$.

We indicate as $C(\mathcal{M})$ the push-forward distribution $\left.\sum_{M \mapsto \mathcal{M}}\{C(M)]\right\}$ induced by a context $C$, and as $\|\mathcal{M}\|$ the norm $\sum_{M \mapsto \mathcal{M}} 1$ of $\mathcal{M}$. Remark, finally, that we have the useful equality $\mathcal{M}=\sum_{M \mapsto \mathcal{M}}\{M\}$. More generally, the sum notation as defined here satisfies some familiar identities, e.g.,

$$
\sum_{N \mapsto\left(\sum_{M \mapsto \mathcal{M}} \mathcal{N}_{M}\right)} \mathcal{L}_{N}=\sum_{M \mapsto \mathcal{M}}\left(\sum_{N \mapsto \mathcal{N}_{M}} \mathcal{L}_{N}\right) .
$$

Example 2.2. If $\mathcal{M}$ is the exponential distribution from Example 2.1 above, and $C$ is the context $(\lambda x . x)(\cdot)$, then the push-forward distribution $C(\mathcal{M})$ is the distribution assigning

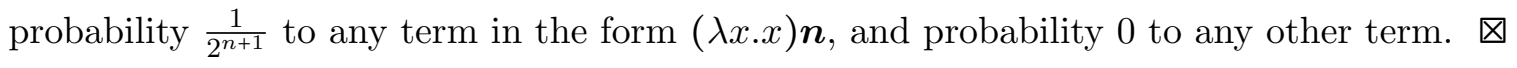




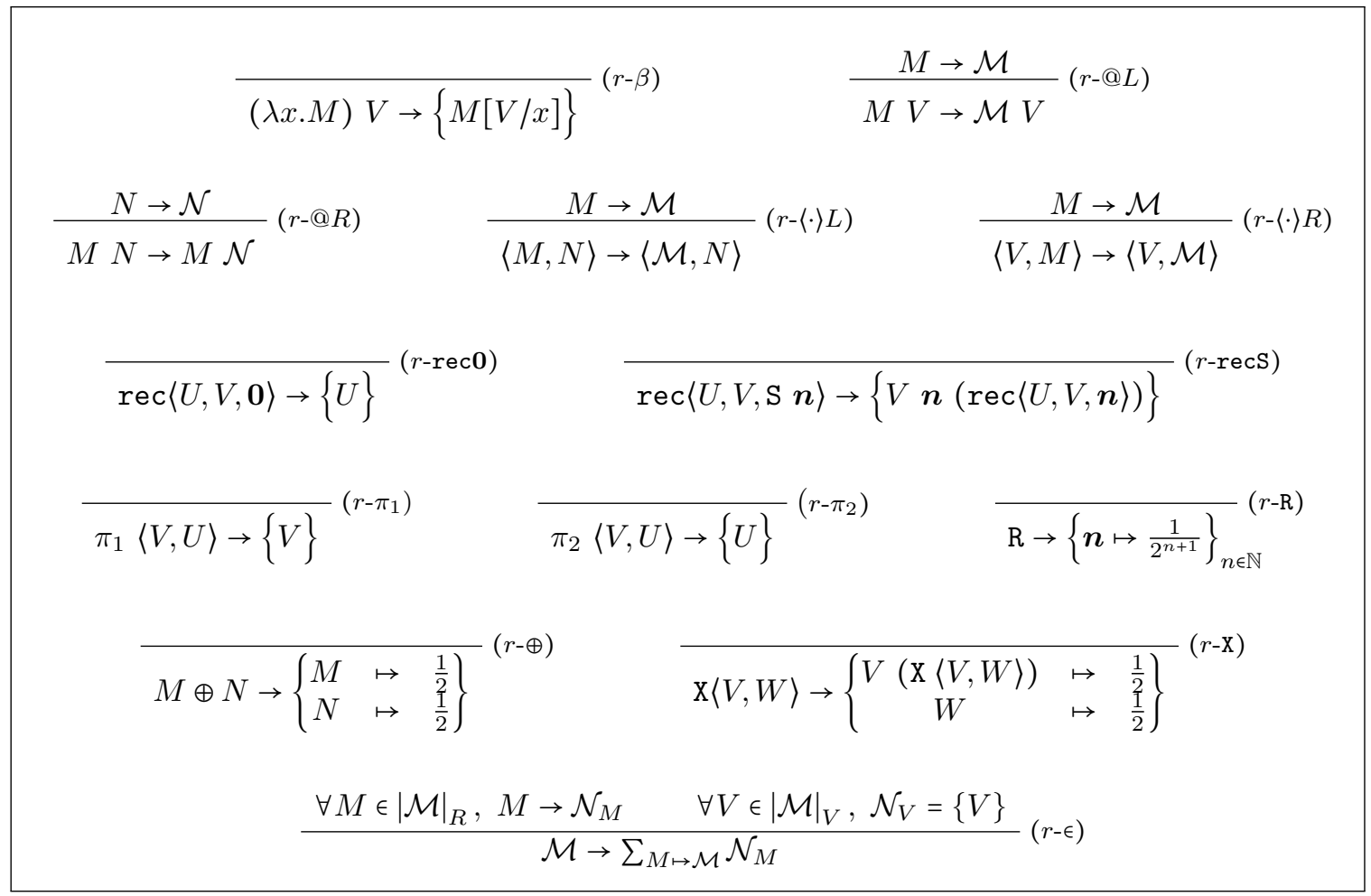

Figure 3. Operational Semantics.

In the following we will often, by abuse of notation, write push-forward distributions like the distribution $C(\mathcal{M})$ from Example 2.2 as $(\lambda x . x) \mathcal{M}$. We even go beyond that, and write expressions like, e.g., $\mathcal{M N}$, which stands for the following distribution:

$$
(\mathcal{M N})(M)= \begin{cases}\mathcal{M}(N) \mathcal{N}(L) & \text { if } M=N L \\ 0 & \text { otherwise }\end{cases}
$$

Reduction rules of $\mathbb{T}^{\oplus, \mathrm{R}, \mathrm{X}}$ are given by Figure 3 . For simplicity, we use the notation $M \rightarrow^{?} \mathcal{M}$ for $\{M\} \rightarrow \mathcal{M}$ (i.e., $M \rightarrow \mathcal{M}$ ) whenever $M$ is reducible and $\mathcal{M}=\{M\}$ whenever $M$ is a value. The sum notation allows us to rewrite rule $(r-\epsilon)$ as a form of monadic lifting:

$$
\frac{\forall M \in|\mathcal{M}|, M \rightarrow^{?} \mathcal{N}_{M}}{\mathcal{M} \rightarrow \sum_{M \mapsto \mathcal{M}} \mathcal{N}_{M}}(r-\epsilon)
$$

Example 2.3. Notice that we can faithfully simulate system $\mathbb{T}$ inside $\mathbb{T}^{\oplus, \mathrm{R}, \mathrm{X}}$. For example, consider the following term:

$$
\text { Expo := } \lambda n . r e c\langle\mathbf{1}, \lambda x y . r e c\langle\mathbf{0}, \lambda x . \mathrm{SS}, y\rangle, \mathrm{S} n\rangle: \mathrm{NAT} \rightarrow \mathrm{NAT}
$$

where SS is a shortcut for the term $\lambda z . \mathrm{S}(\mathrm{S} z)$ computing the double-successor of its argument. This term computes the function $n \mapsto 2^{n+1}$ in time $O\left(2^{n}\right)$. Indeed, when applied to a natural number $\boldsymbol{n}$, it will get the following reduction where we denote 


$$
\begin{aligned}
E_{k}:=\operatorname{rec}\langle\mathbf{1}, \lambda x y \cdot \operatorname{rec}\langle\mathbf{0}, \lambda x . \mathrm{SS}, y\rangle, \boldsymbol{k}\rangle: & \\
(\lambda n . \operatorname{rec}\langle\mathbf{1}, \lambda x y . \operatorname{rec}\langle\mathbf{0}, \lambda x . \mathrm{SS}, y\rangle, \mathrm{S} n\rangle) \boldsymbol{n} & \rightarrow\left\{E_{n+1}\right\} \rightarrow\left\{(\lambda x y \cdot \operatorname{rec}\langle\mathbf{0}, \lambda x . \mathrm{SS}, y\rangle) \boldsymbol{n} E_{n}\right\} \\
& \rightarrow \ldots \rightarrow\left\{\operatorname{rec}\left\langle\mathbf{0}, \lambda x . \mathrm{SS}, \mathbf{2}^{\boldsymbol{n}}\right\rangle\right\} \\
& \rightarrow \ldots \rightarrow\left\{\mathbf{2}^{\boldsymbol{n}+\mathbf{1}}\right\} .
\end{aligned}
$$

Notice that we are only considering Dirac distributions since reduction is deterministic: no probabilistic choice operator occurs in Expo.

Example 2.4. As a second example, we are presenting the term $\mathrm{X}\langle\mathrm{S}, \mathbf{0}\rangle$, whose reduction is essentially probabilistic:

$\mathrm{X}\langle\mathrm{S}, \mathbf{0}\rangle \rightarrow\left\{\begin{array}{cll}\mathrm{S}(\mathrm{X}\langle\mathrm{S}, \mathbf{0}\rangle) & \mapsto & \frac{1}{2} \\ \mathbf{0} & \mapsto & \frac{1}{2}\end{array}\right\} \rightarrow\left\{\begin{array}{ccc}\mathrm{SS}(\mathrm{X}\langle\mathrm{S}, \mathbf{0}\rangle) & \mapsto & \frac{1}{4} \\ \mathbf{1} & \mapsto & \frac{1}{4} \\ \mathbf{0} & \mapsto & \frac{1}{2}\end{array}\right\} \rightarrow\left\{\begin{array}{ccc}\mathrm{SSS}(\mathrm{X}\langle\mathrm{S}, \mathbf{0}\rangle) & \mapsto & \frac{1}{8} \\ \mathbf{2} & \mapsto & \frac{1}{8} \\ \mathbf{1} & \mapsto & \frac{1}{4} \\ \mathbf{0} & \mapsto & \frac{1}{2}\end{array}\right\} \rightarrow \cdots$

Notice that, after $n+1$ reduction steps, the support of the underlying distribution has precisely $n$ elements.

We can easily define $\rightarrow^{n}$ as the $n^{\text {th }}$ iteration of $\rightarrow$ and $\rightarrow^{*}$ as the reflexive and transitive closure of $\rightarrow$. If $\mathcal{M} \rightarrow^{n} \mathcal{N}$ and $\mathcal{U}=\mathcal{N}^{V}$, then we write $\mathcal{M} \rightarrow^{\leq n} \mathcal{U}$. In other words, $\mathcal{U}$ is the distribution on values to which $\mathcal{M}$ reduces in at most $n$ steps. For every $\mathcal{M}$ and for every natural number $n$, there are unique $\mathcal{N}$ and $\mathcal{U}$ such that $\mathcal{M} \rightarrow^{n} \mathcal{N}$ and $\mathcal{M} \rightarrow^{\leq n} \mathcal{U}$.

In probabilistic systems, we might want to consider infinite reduction sequences such as the ones induced by $\mathrm{X}\langle(\lambda x . x), \mathbf{0}\rangle$, which reduces to $\{\mathbf{0}\}$, but only after an infinite number of steps. Please note that for any value $V$, and whenever $\mathcal{M} \rightarrow \mathcal{N}$, it holds that $\mathcal{M}(V) \leq \mathcal{N}(V)$. As a consequence, we can finally give the following definition, one of the most crucial ones in this paper:

Definition 2.5. Let $M$ be a term and let $\left(\mathcal{M}_{n}\right)_{n \in \mathbb{N}}$ be the unique distribution family such that $M \rightarrow^{\leq n} \mathcal{M}_{n}$. The evaluation of $M$ is the value distribution

$$
\llbracket M \rrbracket:=\left\{V \mapsto \lim _{n \rightarrow \infty} \mathcal{M}_{n}(V)\right\} \in \mathfrak{D}\left(\mathbb{T}_{V}^{\oplus, \mathrm{R}, \mathrm{X}}\right) .
$$

The success of $\mathcal{M}$ is its probability of termination, which is formally defined as the norm of its evaluation, i.e., $\operatorname{Succ}(M):=\|\llbracket M \rrbracket\|$. The average reduction length from $M$ is

$$
[M]:=\sum_{n}\left\|\mathcal{M}_{n}^{R}\right\| \in \mathbb{N} \cup\{+\infty\}
$$

The operator $\llbracket \cdot \rrbracket$ can be easily generalized to one on distributions of terms, since the sequence of distributions $\mathcal{M}_{n}$ is anyway unique. Whenever $\llbracket M \rrbracket=\{N\}$, it makes sense to consider $N$ as the normal form of $M$; indeed, we write $N=\mathbb{N F}(M)$ in all these cases, e.g. when $M$ is a term from $\mathbb{T}$.

Example 2.6. Take as an example the term $\mathrm{X}\langle\mathrm{S}, \mathbf{0}\rangle$ from Example 2.4. We have that, for all $n$ :

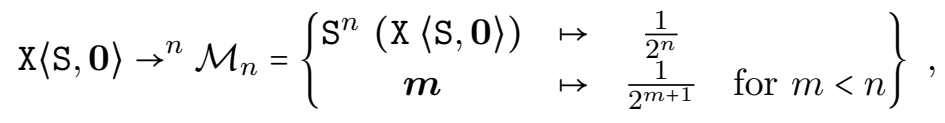


so that $\mathcal{M}_{n}(\boldsymbol{m})=\mathbf{0}$ if $m \geq n$ and $\mathcal{M}_{n}(\boldsymbol{m})=\frac{1}{2^{m+1}}$ otherwise. Thus:

$$
\begin{aligned}
\llbracket \mathrm{X}\langle\mathrm{S}, \mathbf{0}\rangle \rrbracket & =\left\{\boldsymbol{m} \mapsto \lim _{n \rightarrow \infty} \mathcal{M}_{n}(\boldsymbol{m}) \mid m \in \mathbb{N}\right\} \\
& =\left\{\boldsymbol{m} \mapsto \frac{1}{2^{m+1}} \mid m \in \mathbb{N}\right\} .
\end{aligned}
$$

Notice that, by Rule $(r-\epsilon)$, evaluation is continuous:

$$
\llbracket \mathcal{M} \rrbracket=\sum_{M \mapsto \mathcal{M}} \llbracket M \rrbracket
$$

Which kind of mathematical object does any term $M$ of type NAT $\rightarrow$ NAT compute? Following, e.g., [DLZG14], we can say that any such term represents a function $g: \mathbb{N} \rightarrow \mathfrak{D}(\mathbb{N})$ iff for every $m, n$ it holds that $g(n)(m)=\llbracket M \boldsymbol{n} \rrbracket(\boldsymbol{m})$. This will be a key notion not only to evaluate the expressive power of various fragments of $\mathbb{T}^{\oplus, \mathrm{R}, \mathrm{X}}$, but also to compare them.

2.2. On the Continuity of the Operational Semantics. In this section, we will ask ourselves whether the semantics $\llbracket M N \rrbracket$ of an application $M N$ can somehow be given from the semantics of $\llbracket M \rrbracket$ and $\llbracket N \rrbracket$. Lemma 2.11 below gives a positive answer to this question, but some auxiliary lemmas are necessary beforehand.

The first such lemma states that the (one-step) reduction of a sum is the sum of the one-step reducts of its addends. Of course, the different reductions of the addends can have interpolations which makes the decomposition nontrivial:

Lemma 2.7. For every $\mathcal{M}$ and $\left(\mathcal{N}_{M}\right), \sum_{M \mapsto \mathcal{M}} \mathcal{N}_{M} \rightarrow \mathcal{L}$ iff $\mathcal{L}$ can be written as the sum $\sum_{M \mapsto \mathcal{M}} \mathcal{L}_{M}$, where $\mathcal{N}_{M} \rightarrow \mathcal{L}_{M}$ for any $M \in|\mathcal{M}|$.

Proof. Let us analyse the two implications separately:

- Let $\left(\mathcal{L}_{M}\right)_{M \epsilon|\mathcal{M}|}$ such that $\mathcal{N}_{M} \rightarrow \mathcal{L}_{M}$ for any $M \in|\mathcal{M}|$. Then in order to derive $\mathcal{N}_{M} \rightarrow \mathcal{L}_{M}$, the only rule we can apply is $(r-\epsilon)$ so that there is $\left(\mathcal{Q}_{M, N}\right)_{M \in|\mathcal{M}|, N \in\left|\mathcal{N}_{M}\right|}$ such that $\mathcal{L}_{M}=\sum_{N \mapsto \mathcal{N}_{M}} \mathcal{Q}_{M, N}$ and $N \rightarrow^{?} \mathcal{Q}_{M, N}$. Notice that for $N \in\left|\mathcal{N}_{M}\right| \cap\left|\mathcal{N}_{L}\right|$, the determinism of $\rightarrow^{\text {? }}$ gives that $\mathcal{Q}_{M, N}=\mathcal{Q}_{L, N}$, thus we can define $\mathcal{Q}_{N}$ without ambiguity for any $N \in \bigcup_{M \in|\mathcal{M}|}\left|\mathcal{N}_{M}\right|=\left|\sum_{M \mapsto \mathcal{M}} \mathcal{N}_{M}\right|$. Then we have $N \rightarrow^{?} \mathcal{Q}_{N}$ and, thanks to Equation (2.2),

$$
\sum_{N \mapsto\left(\sum_{M \mapsto \mathcal{M}} \mathcal{N}_{M}\right)} \mathcal{Q}_{N}=\sum_{M \mapsto \mathcal{M}}\left(\sum_{M \mapsto \mathcal{N}_{M}} \mathcal{Q}_{N}\right)=\sum_{M \mapsto \mathcal{M}} \mathcal{L}_{M} .
$$

By rule $(r-\epsilon)$ we get $\sum_{M \mapsto \mathcal{M}} \mathcal{N}_{M} \rightarrow \sum_{M \mapsto \mathcal{M}} \mathcal{L}_{M}$.

- Conversely, assume that $\sum_{M \mapsto \mathcal{M}} \mathcal{N}_{M} \rightarrow \mathcal{L}$. In order to derive it, the only rule we can apply is $(r-\epsilon)$ so that there is $\left(\mathcal{Q}_{N}\right)_{N \epsilon\left|\sum_{M \mapsto \mathcal{M}} \mathcal{N}_{M}\right|}$ such that $\mathcal{L}=\sum_{N \mapsto\left(\sum_{M \mapsto \mathcal{M}} \mathcal{N}_{M}\right)} \mathcal{Q}_{N}=$ $\sum_{M \mapsto \mathcal{M}} \sum_{M \mapsto \mathcal{N}_{M}} \mathcal{Q}_{N}$ and $N \rightarrow^{?} \mathcal{Q}_{N}$. We conclude by setting $\mathcal{L}_{M}:=\sum_{N \mapsto \mathcal{N}_{M}} \mathcal{Q}_{N}$.

The decomposition can of course be iterated to reductions of any length:

Lemma 2.8. For every $\mathcal{M}$ and $\left(\mathcal{N}_{M}\right), \sum_{M \mapsto \mathcal{M}} \mathcal{N}_{M} \rightarrow^{n} \mathcal{L}$, iff $\mathcal{L}$ can be written as $\sum_{M \mapsto \mathcal{M}} \mathcal{L}_{M}$, where $\mathcal{N}_{M} \rightarrow^{n} \mathcal{L}_{M}$ for any $M \in|\mathcal{M}|$.

Proof. By induction on $n$ :

- If $n=0$ then $\mathcal{L}_{M}=\mathcal{N}$.

- Otherwise, $\sum_{M \mapsto \mathcal{M}} \mathcal{N}_{M} \rightarrow \mathcal{Q} \rightarrow^{n-1} \mathcal{L}$. By Lemma 2.7, the first step is possible iff $\mathcal{Q}=\sum_{M \mapsto \mathcal{M}} \mathcal{Q}_{M}$ with $\mathcal{N}_{M} \rightarrow \mathcal{Q}_{M}$ for any $M \in|\mathcal{M}|$. By IH, the remaining steps are then possible iff $\mathcal{L}=\sum_{M \mapsto \mathcal{M}} \mathcal{L}_{M}$ such that $\mathcal{Q}_{M} \rightarrow^{n-1} \mathcal{L}_{M}$ for any $M \in|\mathcal{M}|$. 
Thanks to Lemma 2.8, it is possible to trace back values obtained by reducing an application, as stated in the following, intermediate, lemma:

Lemma 2.9. If $(M N) \rightarrow^{n} \mathcal{L} \geq \mathcal{W}$ for some $\mathcal{W} \in \mathfrak{D}\left(\mathbb{T}_{V}^{\oplus, \mathrm{R}, \mathrm{X}}\right)$, then there are distributions $\mathcal{M}, \mathcal{N}, \mathcal{P} \in \mathfrak{D}\left(\mathbb{T}^{\oplus, \mathrm{R}, \mathrm{X}}\right)$ and $\mathcal{U}, \mathcal{V} \in \mathfrak{D}\left(\mathbb{T}_{V}^{\oplus, \mathrm{R}, \mathrm{X}}\right)$ such that $M \rightarrow^{*} \mathcal{M} \geq \mathcal{U}, N \rightarrow^{*} \mathcal{N} \geq \mathcal{V}$, and $(\mathcal{U} \mathcal{V}) \rightarrow^{*} \mathcal{P} \geq \mathcal{W}$

Proof. This is again an induction on $n$. The base case is trivial. If, instead, $n \geq 1$, we proceed differently depending whether $M$ and $N$ are values or not.

- If $N$ is not a value then $N \rightarrow \mathcal{N}^{\prime}$ and $(M N) \rightarrow\left(M \mathcal{N}^{\prime}\right) \rightarrow^{n-1} \mathcal{L} \geq \mathcal{W}$. Notice that $\left(M \mathcal{N}^{\prime}\right):=\sum_{N^{\prime} \mapsto \mathcal{N}^{\prime}}\left\{M N^{\prime}\right\}$. Using Lemma 2.8 , we can decompose $\mathcal{W}=\sum_{N^{\prime} \mapsto \mathcal{N}^{\prime}} \mathcal{W}_{N^{\prime}}$ and $\mathcal{L}=\sum_{N^{\prime} \mapsto \mathcal{N}^{\prime}} \mathcal{L}_{N^{\prime}}$ in such a way that for any $N^{\prime} \in\left|\mathcal{N}^{\prime}\right|,\left(M N^{\prime}\right) \rightarrow^{\leq n-1} \mathcal{L}_{N^{\prime}} \geq \mathcal{W}_{N^{\prime}}$. By induction we have $N^{\prime} \rightarrow^{*} \mathcal{N}_{N^{\prime}} \geq \mathcal{V}_{N^{\prime}}$ and $M \rightarrow^{*} \mathcal{M}_{N^{\prime}} \geq \mathcal{U}_{N^{\prime}}$ with $\left(\mathcal{U}_{N^{\prime}} \mathcal{V}_{N^{\prime}}\right) \rightarrow^{*}$ $\mathcal{P}_{N^{\prime}} \geq \mathcal{W}_{N^{\prime}}$ for all $N^{\prime} \in\left|\mathcal{N}^{\prime}\right|$. Summing up we have $\mathcal{M}:=\sum_{N^{\prime} \mapsto \mathcal{N}^{\prime}} \mathcal{M}_{N^{\prime}}, \mathcal{U}:=\sum_{N^{\prime} \mapsto \mathcal{N}^{\prime}} \mathcal{U}_{N^{\prime}}$, $\mathcal{N}:=\sum_{N^{\prime} \mapsto \mathcal{N}^{\prime}} \mathcal{N}_{N^{\prime}}$ and $\mathcal{V}:=\sum_{N^{\prime} \mapsto \mathcal{N}^{\prime}} \mathcal{V}_{N^{\prime}}$

- If $N$ is a value and $M \rightarrow \mathcal{M}^{\prime}$ then $(M N) \rightarrow\left(\mathcal{M}^{\prime} N\right) \rightarrow^{n-1} \mathcal{L} \geq \mathcal{W}$. Thus we can decompose the equation similarly along $\mathcal{M}^{\prime}$ and apply our IH.

- If both $M$ and $N$ are values it is trivial since $\mathcal{U}=\{M\}$ and $\mathcal{V}=\{N\}$.

We now have all the necessary ingredients for at least proving a restricted form of our desired continuity result:

Lemma 2.10. For every $m, n \in \mathbb{N}$ and every $\mathcal{M}, \mathcal{N} \in \mathfrak{D}\left(\mathbb{T}^{\oplus, \mathrm{R}, \mathrm{X}}\right)$, whenever $M \rightarrow^{m} \mathcal{M}$ and $N \rightarrow^{n} \mathcal{N} \geq \mathcal{V}$, we have $\llbracket M N \rrbracket \geq \llbracket \mathcal{M} \mathcal{V} \rrbracket$. In particular, if $\mathcal{M} \geq \mathcal{U}$, then $\llbracket M N \rrbracket \geq \llbracket \mathcal{U} \mathbb{V} \rrbracket$.

Proof. By induction on $m+n$. The base case $m+n=0$ is trivial. If $n \geq 1$, we proceed differently depending whether $M$ and $N$ are values or not.

- If $N \rightarrow \mathcal{L} \rightarrow^{n-1} \mathcal{N} \geq \mathcal{V}$ then $N$ is not a value. By Lemma 2.8, we can decompose $\mathcal{N}=\sum_{L \mapsto \mathcal{L}} \mathcal{N}_{L}$ and $\mathcal{V}=\sum_{L \mapsto \mathcal{L}} \mathcal{V}_{L}$ in such a way that for any $L \in|\mathcal{L}|, L^{\leq n-1} \mathcal{N}_{L} \geq \mathcal{V}_{L}$ (with $\rightarrow^{\leq n-1}$ standing for either $=$ or $\rightarrow^{n-1}$ depending whether $L$ is a value or not). Then we get

$$
\llbracket M N \rrbracket=\llbracket M \mathcal{L} \rrbracket=\sum_{L \mapsto \mathcal{L}} \llbracket M L \rrbracket \geq \sum_{L \mapsto \mathcal{L}} \llbracket \mathcal{M} \mathcal{V}_{L} \rrbracket=\llbracket \mathcal{M}\left(\sum_{L \mapsto \mathcal{L}} \mathcal{V}_{L}\right) \rrbracket=\llbracket \mathcal{M} \mathcal{V} \rrbracket .
$$

- If $\mathcal{V}$ is the empty (sub)distribution, this is trivial.

- If $n=0$ and $\mathcal{V}$ is not empty, then $\mathcal{V}=\{N\}$, thus $N$ is a value and $M \rightarrow \mathcal{L} \rightarrow^{m-1} \mathcal{M}$, then we can decompose the equation similarly along $\mathcal{L}$ and apply our IH.

The following is a crucial intermediate step towards Theorem 2.13, the main result of this section.

Lemma 2.11. For any $M, N$ it holds that $\llbracket M N \rrbracket=\llbracket \llbracket M \rrbracket \llbracket N \rrbracket \rrbracket$. In particular, if the application $M N$ is almost-surely terminating, so are $M$ and $N$.

Proof. ( $\leq)$ There is $\left(\mathcal{L}_{n}, \mathcal{W}_{n}\right)_{n \geq 1}$ such that $(M N) \rightarrow^{n} \mathcal{L}_{n} \geq \mathcal{W}_{n}$ for all $n$ and such that $\llbracket M N \rrbracket=\lim _{n}\left(\mathcal{W}_{n}\right)$. Applying Lemma 2.9 gives $\mathcal{M}_{n}, \mathcal{N}_{n}, \mathcal{Q}_{n} \in \mathfrak{D}\left(\mathbb{T}^{\oplus, \mathrm{R}, \mathrm{X}}\right)$ and $\mathcal{U}_{n}, \mathcal{V}_{n} \in \mathfrak{D}\left(\mathbb{T}_{V}^{\oplus, \mathrm{R}, \mathrm{X}}\right)$ such that $M \rightarrow^{*} \mathcal{M}_{n} \geq \mathcal{U}_{n}, N \rightarrow^{*} \mathcal{N}_{n} \geq \mathcal{V}_{n}$ and $\left(\mathcal{U}_{n} \mathcal{V}_{n}\right) \rightarrow^{*} \mathcal{Q}_{n} \geq \mathcal{W}_{n}$. Thus $\lim _{n} \mathcal{U}_{n} \leq \llbracket M \rrbracket$ and $\lim _{n} \mathcal{V}_{n} \leq \llbracket N \rrbracket$ with $\mathcal{W}_{n} \leq \llbracket \mathcal{U}_{n} \mathcal{V}_{n} \rrbracket$ leading to the required inequality:

$$
\llbracket M N \rrbracket=\lim _{n} \mathcal{W}_{n} \leq \lim _{n} \llbracket \mathcal{U}_{n} \mathcal{V}_{n} \rrbracket \leq \llbracket \lim _{n}\left(\mathcal{U}_{n} \mathcal{V}_{n}\right) \rrbracket \leq \llbracket\left(\lim _{n} \mathcal{U}_{n}\right)\left(\lim _{n} \mathcal{V}_{n}\right) \rrbracket \leq \llbracket \llbracket M \rrbracket \llbracket N \rrbracket \rrbracket .
$$


( $\geq$ ) There is $\left(\mathcal{M}_{n}, \mathcal{N}_{n}, \mathcal{U}_{n}, \mathcal{V}_{n}\right)_{n \geq 1}$ such that $M \rightarrow^{n} \mathcal{M}_{n} \geq \mathcal{U}_{n}$ and $N \rightarrow^{n} \mathcal{N}_{n} \geq \mathcal{V}_{n}$ for all $n$ and such that $\llbracket M \rrbracket=\lim _{n}\left(\mathcal{U}_{n}\right)$ and $\llbracket N \rrbracket=\lim _{n}\left(\mathcal{V}_{n}\right)$. This leads to the equality $\llbracket \llbracket M \rrbracket \llbracket N \rrbracket \rrbracket=\lim _{m, n} \llbracket \mathcal{U}_{m} \mathcal{V}_{n} \rrbracket$. Finally, by Lemma 2.10 , for any $m, n$, each approximant of $\llbracket \mathcal{U}_{m} \mathcal{V}_{n} \rrbracket$ is below $\llbracket M N \rrbracket$, so is their sup.

2.3. Almost-Sure Termination. We now have all the necessary ingredients to specify a quite powerful notion of probabilistic computation. When, precisely, should such a process be considered terminating? Do all probabilistic branches (see figures 1a-1d) need to be finite? Should we stay more liberal? The literature on the subject is unanimously pointing to a notion called almost-sure termination: a probabilistic computation should be considered terminating if the set of infinite computation branches, although not necessarily empty, has null probability [MM05, FH15, KK15]. This has the following incarnation in our setting:

Definition 2.12. A term $M$ is said to be almost-surely terminating (AST) iff its probability of convergence is maximal, namely if $\operatorname{Succ}(M)=1$.

This section is concerned with proving that $\mathbb{T}^{\oplus, R, \mathrm{X}}$ indeed guarantees almost-sure termination. This will be done by adapting Girard-Tait's reducibility technique to a probabilistic operational semantics.

Theorem 2.13. The full system $\mathbb{T}^{\oplus, \mathrm{R}, \mathrm{X}}$ is almost-surely terminating (AST), i.e., for every $M \in \mathbb{T}^{\oplus, \mathrm{R}, \mathrm{X}}$ it holds that $\operatorname{Succ}(M)=1$.

Proof. The proof is is based on the the notion of a reducible term, which is given as follows by induction on the structure of types:

$$
\begin{aligned}
\operatorname{Red}_{\mathrm{NAT}} & :=\left\{M \in \mathbb{T}_{C}^{\oplus, \mathrm{R}, \mathrm{X}}(\mathrm{NAT}) \mid M \text { is AST }\right\} \\
\operatorname{Red}_{A \rightarrow B} & :=\left\{M \in \in \mathbb{T}_{C}^{\oplus, \mathrm{R}, \mathrm{X}}(A \rightarrow B) \mid \forall V \in \operatorname{Red}_{A} \cap \mathbb{T}_{V}^{\oplus, \mathrm{R}, \mathrm{X}}, M V \in \operatorname{Red}_{B}\right\} \\
\operatorname{Red}_{A \times B} & :=\left\{M \in \in \mathbb{T}_{C}^{\oplus, \mathrm{R}, \mathrm{X}}(A \times B) \mid\left(\pi_{1} M\right) \in \operatorname{Red}_{A},\left(\pi_{2} M\right) \in \operatorname{Red}_{B}\right\}
\end{aligned}
$$

Then we can observe that:

(1) The reducibility candidates over $\operatorname{Red}_{A}$ are $\rightarrow$-saturated. By an induction on $A$ we can indeed show that if $M \rightarrow \mathcal{M}$ then $|\mathcal{M}| \subseteq \operatorname{Red}_{A}$ iff $M \in \operatorname{Red}_{A}$.

- Trivial for $A=$ NAT.

- If $A=B \rightarrow C$, then for all values $V \in \operatorname{Red}_{B},(M V) \rightarrow(\mathcal{M} V)$, thus by IH $(M V) \epsilon$ $\operatorname{Red}_{C}$ iff $|\mathcal{M} V|=\{N V|N \in| \mathcal{M} \mid\} \subseteq \operatorname{Red}_{C}$; which exactly means that $|\mathcal{M}| \subseteq \operatorname{Red}_{B \rightarrow C}$ iff $M \in \operatorname{Red}_{B \rightarrow C}$.

- If $A=A_{1} \times A_{2}$ : then for $i \in\{1,2\},\left(\pi_{i} M\right) \rightarrow\left(\pi_{i} \mathcal{M}\right)$ so that by IH, $\left(\pi_{i} M\right) \in \operatorname{Red}_{A_{i}}$ iff $\left(\pi_{i} \mathcal{M}\right) \subseteq \operatorname{Red}_{A_{i}}$; which exactly means that $|\mathcal{M}| \subseteq \operatorname{Red}_{A_{1} \times A_{2}}$ iff $M \in \operatorname{Red}_{A_{1} \times A_{2}}$.

(2) The reducibility candidates over Red ${ }_{A}$ are precisely the AST terms $M$ such that $\llbracket M \rrbracket \subseteq$ $\left|\operatorname{Red}_{A}\right|$. This goes by induction on $A$.

- Trivial when $A=$ NAT.

- Suppose $A=B \rightarrow C$. Let $M \in \operatorname{Red}_{B \rightarrow C}$. Remark that there is a value $V \in \operatorname{Red}_{B}$, thus $(M V) \in \operatorname{Red}_{C}$ and $(M V)$ is AST by IH; using Lemma 2.11 we get $M$ AST and it is easy to see that if $U \in|\llbracket M \rrbracket|$ then $U \in|\mathcal{M}|$ for some $M \rightarrow^{*} \mathcal{M}$ so that $U \in \operatorname{Red}_{B \rightarrow C}$ by saturation. Conversely, let $M$ be AST with $|\llbracket M \rrbracket| \subseteq \operatorname{Red}_{B \rightarrow C}$ and let $V \in \operatorname{Red}_{B}$ be a value. By $\mathrm{IH}$, for any $U \in|\llbracket M \rrbracket| \subseteq \operatorname{Red}_{B \rightarrow C}$ we have $(U V)$ AST with an evaluation supported by elements of $\operatorname{Re}_{C}$; by Lemma $2.11 \llbracket M V \rrbracket=\llbracket \llbracket M \rrbracket V \rrbracket$ meaning that 
$(M V)$ is AST and has an evaluation supported by elements of $\operatorname{Red}_{C}$, so that we can conclude by IH.

- Suppose $A=A_{1} \times A_{2}$. Let $M \in \operatorname{Red}_{A_{1} \times A_{2}}$. then $\left(\pi_{1} M\right) \in \operatorname{Red}_{A_{1}}$ and $\left(\pi_{1} M\right)$ is AST by IH; using Lemma 2.11 we get $M$ AST and it is easy to see that if $U \in|\llbracket M \rrbracket|$ then $U \in|\mathcal{M}|$ for some $M \rightarrow^{*} \mathcal{M}$ so that $U \in \operatorname{Red}_{A_{1} \rightarrow A_{2}}$ by saturation. Conversely, let $M$ be AST with $|\llbracket M \rrbracket| \subseteq \operatorname{Red}_{A_{1} \rightarrow A_{2}}$ and let $i \in\{1,2\}$. By IH, for any $U \in|\llbracket M \rrbracket| \subseteq \operatorname{Red}_{A_{1} \rightarrow A_{2}}$ we have $\left(\pi_{i} U\right)$ AST with an evaluation supported by elements of $\operatorname{Re} d_{A_{i}}$; by Lemma $2.11 \llbracket \pi_{i} M \rrbracket=\llbracket \pi_{i} \llbracket M \rrbracket \rrbracket$ meaning that $\left(\pi_{i} M\right)$ is AST and has an evaluation supported by elements of $R e d_{A_{i}}$, so that we can conclude by IH.

(3) Every term $M$ such that $x_{1}: A_{1}, \ldots, x_{n}: A_{n} \vdash M: B$ is a candidate in the sense that if $V_{i} \in \operatorname{Red}_{A_{i}}$ for every $1 \leq i \leq n$, then $M\left[V_{1} / x_{1}, \ldots, V_{n} / x_{n}\right] \in \operatorname{Red}_{B}$. This one goes by induction on the type derivation. The only difficult cases are the recursion, the application, the binary choice $\oplus$ and the denumerable choice $\mathrm{X}$ :

- For the operator rec: We have to show that if $U \in \operatorname{Red}_{A}$ and $V \in \operatorname{Red}_{\mathrm{NAT} \rightarrow A \rightarrow A}$ then for all $n \in \mathbb{N},(\operatorname{rec}\langle U, V, \boldsymbol{n}\rangle) \in \operatorname{Red}_{A}$. We proceed by induction on $n$ :

- If $n=0: \operatorname{rec}\langle U, V, \mathbf{0}\rangle \rightarrow\{U\} \subseteq \operatorname{Red}_{A}$ and we conclude by saturation.

- Otherwise: $\operatorname{rec}\langle U, V,(\boldsymbol{n}+\mathbf{1})\rangle \rightarrow V \boldsymbol{n}(\operatorname{rec}\langle U, V, \boldsymbol{n}\rangle) \in \operatorname{Red}_{A}$ since $(\operatorname{rec}\langle U, V, \boldsymbol{n}\rangle) \epsilon$ $\operatorname{Red}_{A}$ by IH and since $\boldsymbol{n} \in \operatorname{Re} d_{\mathbb{N}}$ and $V \in \operatorname{Red}_{\mathbb{N} \rightarrow A \rightarrow A}$, we conclude by saturation.

- For the application: we have to show that if $M \in \operatorname{Red}_{A \rightarrow B}$ and $N \in \operatorname{Red}_{A}$ then $(M N) \in \operatorname{Red}_{B}$. But since $N \in \operatorname{Red}_{A}$, this means that it is AST and for every $V \in|\llbracket N \rrbracket|,(M V) \in \operatorname{Red}_{B}$. In particular, by Lemma 2.11, we have $\llbracket M N \rrbracket=\llbracket M \llbracket N \rrbracket \rrbracket$ so that $(M N)$ is $\mathrm{AST}$ and $|\llbracket M N \rrbracket| \subseteq \cup_{V \in|\llbracket N \rrbracket|}|\llbracket M V \rrbracket| \subseteq \operatorname{Red}_{B}$.

- For the operator $\oplus$ : If $M, N \in \operatorname{Red}_{A}$ then $\left|\left\{\begin{array}{c}M \mapsto \frac{1}{2} \\ N \mapsto \frac{1}{2}\end{array}\right\}\right| \subseteq \operatorname{Red}_{A}$, and, by $\rightarrow$-saturation, $(M \oplus N) \in \operatorname{Red}_{A}$.

- For the operator X: we have to show that for any value $U \in \operatorname{Red}_{A \rightarrow A}$ and $V \in \operatorname{Re} d_{A}$ if holds that $(\mathrm{x} U V) \in \operatorname{Red}_{A}$.

By an easy induction on $n,\left(U^{n} V\right) \in \operatorname{Red}_{A}$ since $U^{0} V=V \in \operatorname{Red}_{B}$ and $U^{n+1} V=$ $U\left(U^{n} V\right) \in \operatorname{Red}_{B}$ whenever $U^{n} V \in \operatorname{Red}_{B}$ and $U \in \operatorname{Red}_{B \rightarrow B}$. Moreover, by an easy induction on $n$ we have

$$
\llbracket \mathrm{X} U V \rrbracket=\frac{1}{2^{n+1}} \llbracket U^{n}(\mathrm{X} U V) \rrbracket+\sum_{i \leq n} \frac{1}{2^{i+1}} \llbracket U^{i} V \rrbracket .
$$

- Trivial for $n=0$.

- We know that $\llbracket \mathrm{X} U V \rrbracket=\frac{1}{2} \llbracket V \rrbracket+\frac{1}{2} \llbracket V(\mathrm{x} U V) \rrbracket$ and we get $\llbracket V(\mathrm{x} U V) \rrbracket=\llbracket V \llbracket \mathrm{x} U V \rrbracket \rrbracket=$ $\frac{1}{2^{n+1}} \llbracket U^{n+1}(\mathrm{X} U V) \rrbracket+\sum_{i \leq n} \frac{1}{2^{i+1}} \llbracket U^{i+1} V \rrbracket$ by Lemma 2.11 and $\mathrm{IH}$, which is sufficient to conclude.

At the limit, we get $\llbracket \mathrm{X} U V \rrbracket=\sum_{i \in \mathbb{N}} \frac{1}{2^{i+1}} \llbracket U^{i} V \rrbracket$. We can then conclude that $(\mathrm{X} U V$ ) is AST (since each of the $\left(U^{i} V\right) \in \operatorname{Red}_{B}$ are AST and $\sum_{i} \frac{1}{2^{i+1}}=1$ ) and that $|\llbracket M N \rrbracket|=$ $\cup_{i}\left|\llbracket U^{i} V \rrbracket\right| \subseteq \operatorname{Red}_{A}$.

Points 2 and 3 above together leads to the thesis: if $\vdash M: A$, then Point 3 implies that $M \in \operatorname{Red}_{A}$ which, by Point 2, implies that $M$ is AST.

Almost-sure termination could however be seen as too weak a property: there is no guarantee about the average computation length, which can well be infinite even if the probability of termination is finite. For this reason, a stronger notion is often considered, namely positive almost-sure termination: 
Definition 2.14. A term $M$ is said to be positively almost-surely terminating (or PAST) iff the average reduction length $[M]$ is finite.

Gödel's $\mathbb{T}$, when paired with $R$, is combinatorially too powerful to guarantee positive almost sure termination already. This comes from the possibility to describe programs with exponential reduction time such as the term Expo from Example 2.3, which is computing the function $n \mapsto 2^{n+1}$ in time $\Theta\left(2^{n}\right)$.

Theorem 2.15. $\mathbb{T}^{\oplus, \mathrm{R}, \mathrm{X}}$ is not positively almost-surely terminating.

Proof. The term (Expo R) : NAT is computing, with probability $\frac{1}{2^{n+1}}$ the number $2^{n+1}$ in time $\Theta\left(2^{n}\right)$; the average reduction length is thus

$$
\left[\text { Expo R] }=\sum_{n} \frac{\Theta\left(2^{n}\right)}{2^{n+1}}=+\infty\right. \text {. }
$$

Please observe that the counterexample to positive almost-sure termination for $\mathbb{T}^{\oplus, R, X}$ has been obtained by applying Expo to R, and both these terms are positively almost surely terminating when considered in isolation. In other words, positive almost sure termination is not a compositional property.

2.4. On Fragments of $\mathbb{T}^{\oplus, \mathrm{R}, \mathrm{X}}$ : a Roadmap. The calculus $\mathbb{T}^{\oplus, \mathrm{R}, \mathrm{X}}$ contains at least four fragments, namely Gödel's $\mathbb{T}$ and the three fragments $\mathbb{T}^{\oplus}, \mathbb{T}^{\mathrm{R}}$ and $\mathbb{T}^{\mathrm{X}}$ corresponding to the three probabilistic choice operators we consider. It is then natural to ask ourselves how these fragments relate to each other as for their respective expressive power. At the end of this paper, we will have a very clear picture in front of us.

The first such result is the equivalence between the two fragments $\mathbb{T}^{\mathrm{R}}$ and $\mathbb{T}^{\mathrm{X}}$. The embeddings are in fact quite simple: getting $\mathrm{X}$ from $\mathrm{R}$ only requires "guessing" the number of iterations via $R$ and then use rec to execute them; capturing $R$ from $X$ is even easier: it corresponds to counting the total number of iterations performed by $\mathrm{X}$ :

Proposition 2.16. $\mathbb{T}^{\mathrm{R}}$ and $\mathbb{T}^{\mathrm{X}}$ are both equiexpressive with $\mathbb{T}^{\oplus, \mathrm{R}, \mathrm{X}}$.

Proof. The calculus $\mathbb{T}^{\mathrm{R}}$ embeds the full system $\mathbb{T}^{\oplus, \mathrm{R}, \mathrm{X}}$ via the encoding: ${ }^{1}$

$$
M \oplus_{\mathrm{R}} N:=\operatorname{rec}\langle\lambda z \cdot N, \lambda x y z \cdot M, \mathrm{R}\rangle \mathbf{0} ; \quad \mathrm{X}_{\mathrm{R}}:=\lambda x \cdot \operatorname{rec}\left\langle\pi_{2} x, \lambda z \cdot \pi_{1} x, \mathrm{R}\right\rangle .
$$

The fragment $\mathbb{T}^{\mathrm{X}}$ embeds the full system $\mathbb{T}^{\oplus, \mathrm{R}, \mathrm{X}}$ via the encoding:

$$
M \oplus_{\mathrm{X}} N:=\mathrm{X}\langle\lambda x y \cdot M, \lambda y \cdot N\rangle \mathbf{0} ; \quad \mathrm{R}_{\mathrm{X}}:=\mathrm{X}\langle\mathrm{S}, \mathbf{0}\rangle .
$$

In both cases, the embedding is compositional and preserves types. We have to prove the correctness of the two embeddings:

- For any $M$ and $N$ :

$$
\llbracket M \oplus_{\mathrm{R}} N \rrbracket=\llbracket M \oplus_{\mathrm{X}} N \rrbracket=\llbracket M \oplus N \rrbracket=\frac{1}{2} \llbracket M \rrbracket+\frac{1}{2} \llbracket N \rrbracket .
$$

\footnotetext{
${ }^{1}$ Notice that the dummy abstractions on $z$ and the $\mathbf{0}$ at the end ensure the correct reduction order by making $\lambda z . N$ a value.
} 
Indeed, we only have to perform a few reductions:

$$
\begin{aligned}
\llbracket M \oplus_{\mathrm{R}} N \rrbracket & =\sum_{n \geq 0} \frac{1}{2^{n+1}} \llbracket \operatorname{rec}\langle\lambda z \cdot N, \lambda x y z \cdot M, \boldsymbol{n}\rangle \mathbf{0} \rrbracket \\
& =\frac{1}{2} \llbracket \operatorname{rec}\langle\lambda z \cdot N, \lambda x y z \cdot M, \mathbf{0}\rangle \mathbf{0} \rrbracket+\sum_{n \geq 0} \frac{1}{2^{n+2}} \llbracket \operatorname{rec}\langle\lambda z \cdot N, \lambda x y z \cdot M, \mathbf{S} \boldsymbol{n}\rangle \mathbf{0} \rrbracket \\
& =\frac{1}{2} \llbracket(\lambda z \cdot N) \mathbf{0} \rrbracket+\sum_{n \geq 0} \frac{1}{2^{n+2}} \llbracket(\lambda x y z \cdot M) \boldsymbol{n}(\operatorname{rec}\langle\lambda z \cdot N, \lambda x y z \cdot M, \boldsymbol{n}\rangle) \mathbf{0} \rrbracket \\
& =\frac{1}{2} \llbracket N \rrbracket+\sum_{n \geq 0} \frac{1}{2^{n+2}} \llbracket M \rrbracket \\
& =\frac{1}{2} \llbracket N \rrbracket+\frac{1}{2} \llbracket M \rrbracket \\
\llbracket M \oplus_{\mathrm{X}} N \rrbracket & =\frac{1}{2} \llbracket(\lambda y \cdot N) \mathbf{0} \rrbracket+\frac{1}{2} \llbracket(\lambda x y \cdot M) \mathrm{R}_{\mathrm{X}} \mathbf{0} \rrbracket \\
& =\frac{1}{2} \llbracket(\lambda y \cdot N) \mathbf{0} \rrbracket+\frac{1}{2} \llbracket(\lambda x y \cdot M) \mathrm{R}_{\mathrm{X}} \mathbf{0} \rrbracket \\
& =\frac{1}{2} \llbracket N \rrbracket+\frac{1}{2} \llbracket M \rrbracket
\end{aligned}
$$

- For any $U$ and $V$ :

$$
\llbracket \mathrm{X}_{\mathrm{R}}\langle U, V\rangle \rrbracket=\llbracket \mathrm{x}\langle U, V\rangle \rrbracket
$$

Indeed, both of them are the unique fixpoint of the following contractive function:

$$
f(\mathcal{X}):=\frac{1}{2} \llbracket U \rrbracket+\frac{1}{2} \llbracket V \mathcal{X} \rrbracket .
$$

That $\llbracket \mathrm{X}\langle U, V\rangle \rrbracket=f(\llbracket \mathrm{X}\langle U, V\rangle \rrbracket)$ is immediate after a reduction, as for the other, we have:

$$
\begin{aligned}
\llbracket \mathrm{X}_{\mathrm{R}}\langle U, V\rangle \rrbracket & =\sum_{n \geq 0} \frac{1}{2^{n+1}} \llbracket \operatorname{rec}\langle U, \lambda z . V, \boldsymbol{n}\rangle \rrbracket \\
& =\frac{1}{2} \llbracket U \rrbracket+\frac{1}{2} \sum_{n \geq 0} \frac{1}{2^{n+1}} \llbracket U(\operatorname{rec}\langle U, \lambda z \cdot V, \boldsymbol{n}\rangle) \rrbracket \\
& =\frac{1}{2} \llbracket U \rrbracket+\frac{1}{2} \sum_{n \geq 0} \frac{1}{2^{n+1}} \llbracket U \llbracket \operatorname{rec}\langle U, \lambda z \cdot V, \boldsymbol{n}\rangle \rrbracket \rrbracket \\
& =\frac{1}{2} \llbracket U \rrbracket+\frac{1}{2} \llbracket U\left(\sum_{n \geq 0} \frac{1}{2^{n+1}} \llbracket \operatorname{rec}\langle U, \lambda z \cdot V, \boldsymbol{n}\rangle \rrbracket\right) \rrbracket \\
& =\frac{1}{2} \llbracket U \rrbracket+\frac{1}{2} \llbracket U \llbracket \mathrm{X}_{R}\langle U, V\rangle \rrbracket \rrbracket
\end{aligned}
$$

by Lemma 2.11

- Finally:

$$
\llbracket \mathrm{R}_{\mathrm{X}} \rrbracket=\llbracket \mathrm{R} \rrbracket=\left\{\boldsymbol{n} \mapsto \frac{1}{2^{n+1}} \mid n \geq 0\right\} .
$$

That $\llbracket \mathrm{R} \rrbracket=\left\{\boldsymbol{n} \mapsto \frac{1}{2^{n+1}} \mid n \geq 0\right\}$ is just one step of reduction, while $\llbracket \mathrm{R}_{\mathbf{X}} \rrbracket=\left\{\boldsymbol{n} \mapsto \frac{1}{2^{n+1}} \mid n \geq 0\right\}$ was shown in Example 2.6.

Notice how simulating $\mathrm{X}$ by $\mathrm{R}$ requires the presence of recursion, while the converse is not true. The implications of this fact are intriguing, but lie outside the scope of this work. In the following, we will no longer consider $\mathbb{T}^{\mathrm{X}}$ nor $\mathbb{T}^{\oplus, \mathrm{R}, \mathrm{X}}$ but only $\mathbb{T}^{\mathrm{R}}$, keeping in mind that all these are equiexpressive due to Proposition 2.16. The rest of this paper, thus, will be 
concerned with understanding the relative expressive power of the three fragments $\mathbb{T}, \mathbb{T}^{\oplus}$, and $\mathbb{T}^{\mathrm{R}}$. Can any of the (obvious) strict syntactical inclusions between them be turned into a strict semantic inclusion? Are the three systems equiexpressive?

In order to compare probabilistic and deterministic calculi, several options are available. The most common one is to consider notions of observations over the probabilistic outputs; this will be pursued in Section 5, where we look at whether Monte Carlo or Las Vegas algorithms on $\mathbb{T}^{\oplus}$ or $\mathbb{T}^{\mathrm{R}}$ result in deterministically $\mathbb{T}$-definable functions or not. Notice that neither Monte Carlo nor Las Vegas algorithms are natively definable inside $\mathbb{T}^{\oplus, R, X}$. Indeed, those algorithms are based on restrictions on the resulting distribution, which cannot be described in the calculus. For example, a Las Vegas algorithm is captured by, say, a term $M:$ NAT $\rightarrow$ NAT such that $\llbracket M n \rrbracket(0) \leq \frac{1}{2}$ for any $n$. In the next two sections, instead, we look at how $\mathbb{T}^{\oplus, R, X}$ can be seen as a way to compute functions returning distributions over the base type $\mathbb{N}$ rather than elements of if.

We say that the distribution $\mathcal{M} \in \mathfrak{D}(\mathbb{N})$ is finitely represented by ${ }^{2} f: \mathbb{N} \rightarrow \mathbb{B}$, if there exists a natural number $q$ such that for every $k \geq q$ it holds that $f(k)=0$ and $\mathcal{M}=\{\boldsymbol{k} \mapsto f(k)\}$. Moreover, the definition can be extended to families of distributions $\left(\mathcal{M}_{n}\right)_{n}$ by requiring the existence of functions $f: \mathbb{N} \times \mathbb{N} \rightarrow \mathbb{B}$ and $q: \mathbb{N} \rightarrow \mathbb{N}$ such that for every $k \geq q(n)$ it holds that $f(n, k)=0$ and for every $n$ it holds that $\mathcal{M}_{n}=\{\boldsymbol{k} \mapsto f(n, k)\}$. In this case, we say that the representation is parametric. We will see in Section 3 that the distributions computed by $\mathbb{T}^{\oplus}$ are exactly the ones (parametrically) finitely representable by $\mathbb{T}$ terms. Concretely, this means that for any $M \in \mathbb{T}^{\oplus}(\mathrm{NAT})$ or any $M \in \mathbb{T}^{\oplus}(\mathrm{NAT} \rightarrow \mathrm{NAT})$, the distributions $\llbracket M \rrbracket$ and $(\llbracket N \boldsymbol{n} \rrbracket)_{n}$ are (parametrically) finitely representable.

In $\mathbb{T}^{\mathrm{R}}$, however, distributions are more complex, having infinite support and giving rise to non-rational probabilities. That is why only a characterization in terms of approximations is possible. More specifically, a distribution $\mathcal{M} \in \mathfrak{D}(\mathbb{N})$ is said to be approximated by two functions $f: \mathbb{N} \times \mathbb{N} \rightarrow \mathbb{B}$ and $g: \mathbb{N} \rightarrow \mathbb{N}$ iff for every $n \in \mathbb{N}$ and for every $k \geq g(n)$ it holds that $f(n, k)=0$ and

$$
\sum_{k \in \mathbb{N}}|\mathcal{M}(\boldsymbol{k})-f(n, k)| \leq \frac{1}{n}
$$

In other words, the distribution $\mathcal{M}$ can be approximated arbitrarily well, and uniformly, by finitely representable ones. Similarly, we can define a parametric version of this definition at first order. In Section 4 , we show that distributions generated by $\mathbb{T}^{\mathrm{R}}$ terms are indeed uniform limits over those of $\mathbb{T}^{\oplus}$; our result on $\mathbb{T}^{\oplus}$ thus induces their (parametric) approximation in $\mathbb{T}$.

\section{Binary Probabilistic Choice}

This section is concerned with two theoretical results on the expressive power of $\mathbb{T}^{\oplus}$. Taken together, they tell us that this fragment is not far from $\mathbb{T}$.

\footnotetext{
${ }^{2}$ Here we denote $\mathbb{B}$ for binomial numbers $\frac{m}{2^{n}}$ (where $m, n \in \mathbb{N}$ ) and BIN for their representation in system $\mathbb{T}$ encoded by pairs $\langle m, n\rangle$ of natural numbers.
} 


$$
\overline{M \Rightarrow\{M\}}(R-0) \quad \frac{M \rightarrow \mathcal{M} \mathcal{M} \Rightarrow \mathcal{N}}{M \Rightarrow \mathcal{N}}(R \text {-tran }) \quad \frac{\forall M \in|\mathcal{M}|, M \Rightarrow \mathcal{N}_{M}}{\mathcal{M} \Rightarrow \sum_{M \mapsto \mathcal{M}} \mathcal{N}_{M}}(R-\epsilon)
$$

Figure 4. Multistep Reduction.

3.1. Positive Almost-Sure Termination. As we already observed, the average number of steps to normal form can be infinite for terms of $\mathbb{T}^{\oplus, R, X}$. We will prove that, on the contrary, $\mathbb{T}^{\oplus}$ is positive almost-surely terminating. This will be done by adapting (and strengthening!) the reducibility-based result from Section 2.3. To this end, we will first give a formalization of the notion of execution tree discussed in Section 1 in the form of a multistep reduction procedure. Then, we will formally show that this tree is finite. We will see later that the multistep reduction is nothing more than $\rightarrow^{*}$ for $\mathbb{T}^{\oplus}$, but that this is not the case in richer fragments of $\mathbb{T}^{\oplus, R, X}$.

Definition 3.1. The multistep reduction relation $\Rightarrow$ is defined by induction in Figure 4 . Due to the (potentially) countably many preconditions of the rule $(R-\epsilon)$, the derivation tree of a multistep reduction $\Rightarrow$ can be infinitely wide and even of unbounded height, but each path have to be finite.

The infiniteness of the width and the fact that the height is unbounded is an essential tool for analising $\mathbb{T}^{R}$. In fact, most theorems in this section will be given for both $\mathbb{T}^{\oplus}$ and $\mathbb{T}^{\mathrm{R}}$. But, for now, we will focus on $\mathbb{T}^{\oplus}$ and finite derivations, while $\mathbb{T}^{R}$ and transfinite derivations will be analyzed in details in Section 4.1.

Example 3.2. Consider the already considered example term $\operatorname{rec}\langle\mathbf{0}, \lambda x y . y \oplus(\mathrm{S} y), \mathbf{2}\rangle$; the execution tree is the following, where $U_{n}$ stands for $\operatorname{rec}\langle\mathbf{0}, \lambda x y . y \oplus(\mathrm{S} y), \boldsymbol{n}\rangle$ :

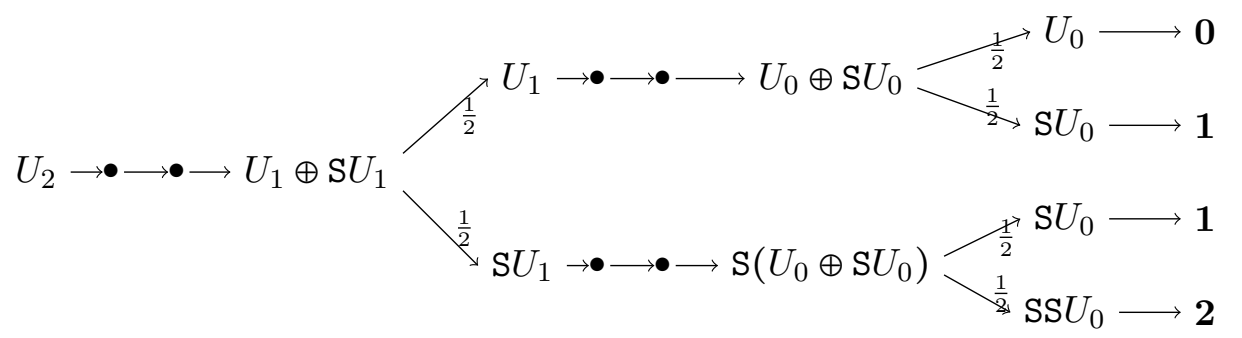

This tree is subsumed by the (more complex) derivation of $U_{2} \Rightarrow \frac{1}{4}\{\mathbf{0}\}+\frac{1}{2}\{\mathbf{1}\}+\frac{1}{4}\{\mathbf{2}\}$, with every arrow of the exectution tree replaced by a $(R$-tran) rule followed by a $(R-\epsilon)$ rule: 


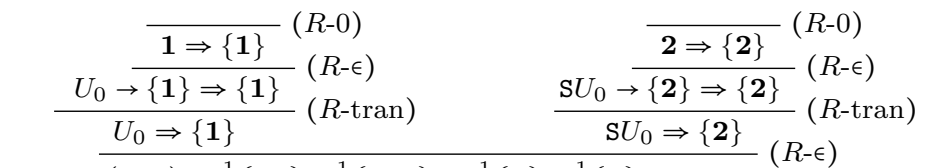

$$
\begin{aligned}
& \frac{U_{0} \oplus \overline{\left(\mathrm{S} U_{0}\right) \rightarrow \frac{1}{2}\left\{U_{0}\right\}+\frac{1}{2}\left\{\mathrm{~S} U_{0}\right\} \Rightarrow \frac{1}{2}\{\mathbf{1}\}+\frac{1}{2}\{\mathbf{2}\}}(R \text {-tran) }}{U_{0} \oplus\left(\mathrm{S} U_{0}\right) \Rightarrow \frac{1}{2}\{\mathbf{1}\}+\frac{1}{2}\{\mathbf{2}\}} \\
& \underline{(\lambda y . y \oplus(\mathrm{S} y)) U_{0} \rightarrow \overline{\left\{U_{0} \oplus\left(\mathrm{S} U_{0}\right)\right\} \Rightarrow \frac{1}{2}\{\mathbf{1}\}+\frac{1}{2}\{\mathbf{2}\}}}(R-\epsilon) \\
& (\lambda y \cdot y \oplus(\mathrm{S} y)) U_{0} \Rightarrow \frac{1}{2}\{\mathbf{1}\}+\frac{1}{2}\{\mathbf{2}\} \\
& \left(\lambda x y . y \oplus(\mathrm{S} y) \mathbf{1} U_{0}\right) \rightarrow \overline{\left\{(\lambda y . y \oplus(\mathrm{S} y)) U_{0}\right\} \Rightarrow \frac{1}{2}\{\mathbf{1}\}+\frac{1}{2}\{\mathbf{2}\}} \\
& U_{1} \rightarrow \frac{(\lambda x y . y \oplus(\mathrm{S} y)) \mathbf{1} U_{0} \Rightarrow \frac{1}{2}\{\mathbf{1}\}+\frac{1}{2}\{\mathbf{2}\}}{\left\{(\lambda x y \cdot y \oplus(\mathrm{S} y)) \mathbf{1} U_{0}\right\} \Rightarrow \frac{1}{2}\{\mathbf{1}\}+\frac{1}{2}\{\mathbf{2}\}}(R-\epsilon) \\
& \frac{U_{1} \rightarrow \overline{\left\{(\lambda x y \cdot y \oplus(\mathrm{S} y)) \mathbf{1} U_{0}\right\} \Rightarrow \frac{1}{2}\{\mathbf{1}\}+\frac{1}{2}\{\mathbf{2}\}}}{U_{1} \Rightarrow \frac{1}{2}\{\mathbf{0}\}+\frac{1}{2}\{\mathbf{1}\}}(R \text { - } \epsilon) \\
& \frac{U_{1} \oplus\left(\mathrm{S} U_{1}\right) \rightarrow \frac{1}{2}\left\{U_{1}\right\}+\frac{1}{2}\left\{\mathrm{~S} U_{1}\right\} \Rightarrow \frac{1}{4}\{\mathbf{0}\}+\frac{1}{2}\{\mathbf{1}\}+\frac{1}{4}\{\mathbf{2}\}}{(R \text {-tran })}(R-\epsilon) \\
& \underline{(\lambda y . y \oplus(\mathrm{S} y)) U_{1} \rightarrow\left\{U_{1} \oplus\left(\mathrm{S} U_{1}\right)\right\} \Rightarrow \frac{1}{4}\{\mathbf{0}\}+\frac{1}{2}\{\mathbf{1}\}+\frac{1}{4}\{\mathbf{2}\}}(R-\epsilon)
\end{aligned}
$$

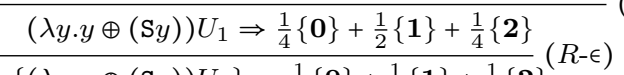

$$
\begin{aligned}
& \frac{(\lambda x y . y \oplus(\mathrm{S} y)) \mathbf{1} U_{1} \rightarrow\left\{(\lambda y . y \oplus(\mathrm{S} y)) U_{1}\right\} \Rightarrow \frac{1}{4}\{\mathbf{0}\}+\frac{1}{2}\{\mathbf{1}\}+\frac{1}{4}\{\mathbf{2}\}}{(R-\epsilon)}(R \text {-tran }) \\
& \frac{\frac{(\lambda x y . y \oplus(\mathrm{S} y)) \mathbf{1} U_{1} \Rightarrow \frac{1}{4}\{\mathbf{0}\}+\frac{1}{2}\{\mathbf{1}\}+\frac{1}{4}\{\mathbf{2}\}}{U_{2} \rightarrow\left\{(\lambda x y \cdot y \oplus(\mathrm{S} y)) \mathbf{1} U_{1}\right\} \Rightarrow \frac{1}{4}\{\mathbf{0}\}+\frac{1}{2}\{\mathbf{1}\}+\frac{1}{4}\{\mathbf{2}\}}(R-\epsilon)}{U_{2} \Rightarrow \frac{1}{4}\{\mathbf{0}\}+\frac{1}{2}\{\mathbf{1}\}+\frac{1}{4}\{\mathbf{2}\}}(R \text {-tran })
\end{aligned}
$$

where $\xi$ is the following derivation:

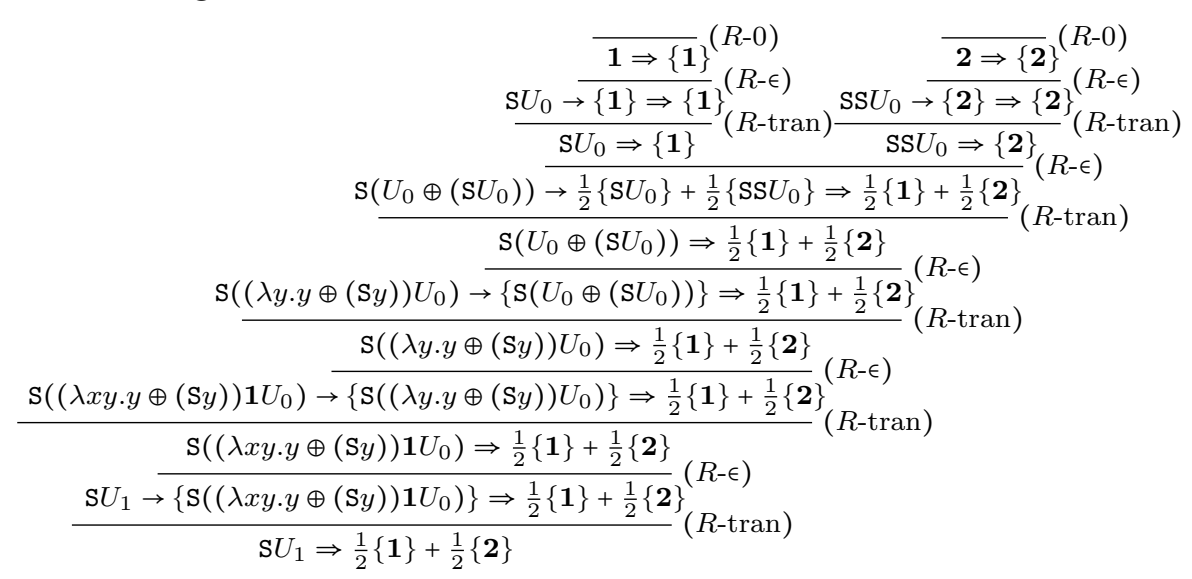

Notice that the derivation is correct and finite because the execution tree is finite.

Lemma 3.3. The multistep semantics $\Rightarrow$ is confluent.

Proof. By an easy induction, we show that if $\mathcal{N} \Leftarrow M \Rightarrow \mathcal{L}$ (resp. $\mathcal{N} \Leftarrow \mathcal{M} \Rightarrow \mathcal{L}$ ), then there is $\mathcal{P}$ such that $\mathcal{N} \Rightarrow \mathcal{P} \Leftarrow \mathcal{L}$. Now:

- If both reductions are using the same rule ( either $(R-0),(R$-tran), or $(R-\epsilon))$, then it is an immediate use of the induction hypothesis on the premises as those rules are determinist.

- If one of them use the rule $(R-0)$, then it is trivial.

- No other case is possible as $(R$-tran) and $(R-\epsilon)$ cannot apply together (one require a term as source and the other a distribution).

Lemma 3.4. If $(M V) \Rightarrow \mathcal{U} \in \mathfrak{D}\left(\mathbb{T}_{V}^{\oplus, \mathrm{R}, \mathrm{X}}\right)$ then there is $\mathcal{W} \in \mathfrak{D}\left(\mathbb{T}_{V}^{\oplus, \mathrm{R}, \mathrm{X}}\right)$ such that $M \Rightarrow \mathcal{W}$. 
Proof. By induction on $\Rightarrow$ we can show that if $(M V) \Rightarrow \mathcal{N}$ then $\mathcal{N}=\mathcal{L}+(\mathcal{M} V)$ with $M \Rightarrow \mathcal{W}+\mathcal{M}$ and $(\mathcal{W} V) \Rightarrow \mathcal{L}$ (and similarly if $M$ is a distribution):

- The $(R-0)$ and $(R-\epsilon)$ are trivial,

- If $(M V) \rightarrow \mathcal{Q} \Rightarrow \mathcal{N}$ then there are two cases:

- Either $M \rightarrow \mathcal{P}$ and $\mathcal{Q}=(\mathcal{P} V) \Rightarrow \mathcal{N}$ and we can conclude by induction hypothesis;

- Or $M=W$ is a value and $M \Rightarrow\{W\}$ with $(\{W\} V) \rightarrow \mathcal{Q} \Rightarrow \mathcal{N}=\mathcal{L}$.

Notice that if $\mathcal{N}$ is a value distribution then $\mathcal{M}$ has to be one.

The following lemma is an alternative version of Lemma 2.11, where the evaluation is obtained after a finite number of steps (in both hypothesis and conclusions).

Lemma 3.5. If $N \Rightarrow \mathcal{V} \in \mathfrak{D}\left(\mathbb{T}_{V}^{\oplus, \mathrm{R}, \mathrm{X}}\right)$ and $M \mathcal{V} \Rightarrow \mathcal{U} \in \mathfrak{D}\left(\mathbb{T}_{V}^{\oplus, \mathrm{R}, \mathrm{X}}\right)$ then $M N \Rightarrow \mathcal{U}$.

Proof. By induction on the derivation of $N \Rightarrow V$ (generalizing the property for any distribution $\mathcal{N}$ in place of $N)$ :

- If $V=\{N\}$ this is trivial.

- If $M \rightarrow \mathcal{N} \Rightarrow \mathcal{V}$ then by IH, $M \mathcal{N} \Rightarrow \mathcal{U}$ and thus $M N \rightarrow M \mathcal{N} \Rightarrow \mathcal{U}$ so that we can conclude by rule $(R-+)$.

- If for all $N \in|\mathcal{N}|, N \Rightarrow \mathcal{V}_{N}$ with $\mathcal{V}=\sum_{N \mapsto \mathcal{N}} \mathcal{V}_{N}$ then by applying the IH on each $N \in|\mathcal{N}|$, we get that $M N \Rightarrow \mathcal{U}_{N}$ for some $\mathcal{U}_{N}$ and we conclude by rule $(R-\epsilon)$ using $\mathcal{U}=\sum_{N \mapsto \mathcal{N}} \mathcal{U}_{N}$.

Theorem 3.6. For any term $M \in \mathbb{T}^{\oplus, \mathrm{R}}$, there exists a value distribution $\llbracket M \rrbracket_{a} \in \mathfrak{D}\left(\mathbb{T}_{V}^{\oplus, \mathrm{R}}\right)$ such that $M \Rightarrow \llbracket M \rrbracket_{a}$. We call it the accessible evaluation.

Proof. When it exists, $\llbracket M \rrbracket_{a}$ is unique due to confluence. Thus we only have to prove its existence. The proof goes by reducibility over reducibility sets defined as follows:

$$
\begin{aligned}
\operatorname{Red}_{\mathrm{NAT}} & :=\left\{M \in \mathbb{T}^{\oplus}(\mathrm{NAT}) \mid \exists \llbracket M \rrbracket_{a} \in \mathfrak{D}\left(\mathbb{T}_{V}^{\oplus}\right), M \Rightarrow \llbracket M \rrbracket_{a}\right\} \\
\operatorname{Red}_{A \rightarrow B} & :=\left\{M \in \mathbb{T}^{\oplus}(A \rightarrow B) \mid \forall V \in \operatorname{Red}_{A} \cap \mathbb{T}_{V}^{\oplus},(M V) \in \operatorname{Red}_{B}\right\} \\
\operatorname{Red}_{A \times B} & :=\left\{M \in \mathbb{T}^{\oplus}(A \times B) \mid\left(\pi_{1} M\right) \in \operatorname{Red}_{A},\left(\pi_{2} M\right) \in \operatorname{Red}_{B}\right\}
\end{aligned}
$$

(1) The reducibility candidates over $\operatorname{Red}_{A}$ are $\rightarrow$-saturated. By induction on $A$ we can show that if $M \rightarrow \mathcal{M}$ then $|\mathcal{M}| \subseteq \operatorname{Red}_{A}$ iff $M \in \operatorname{Red}_{A}$.

- If $A=\mathrm{NAT}$ : then whenever $N \Rightarrow \llbracket N \rrbracket_{a}$ for all $N \in|\mathcal{M}|$ we get $\mathcal{M} \Rightarrow \sum_{N \mapsto \mathcal{M}} \llbracket N \rrbracket_{a}=$ $\llbracket \mathcal{M} \rrbracket_{a}$ by $(R-\epsilon)$ and thus $M \Rightarrow \llbracket \mathcal{M} \rrbracket_{a}=\llbracket M \rrbracket_{a}$ by $(R$-trans $)$. Conversely, whenever $M \Rightarrow \llbracket M \rrbracket_{a}$, this reduction cannot come from rule $(R-0)$ since $\llbracket M \rrbracket_{a}$ is a value distribution and $M$ is reducible, thus it comes from rule $(R-+)$ and $\mathcal{M} \Rightarrow \llbracket \mathcal{M} \rrbracket_{a}$ which itself necessarily comes from an application of $(R-\epsilon)$ so that $N \Rightarrow \llbracket N \rrbracket_{a}$ for any $N \in|\mathcal{M}|$.

- If $A=B \rightarrow C$ : then for all values $V \in \operatorname{Red}_{B},(M V) \rightarrow(\mathcal{M} V)$, thus by IH $(M V) \epsilon$ $\operatorname{Red}_{C}$ iff $|\mathcal{M} V|=\{N V|N \in| \mathcal{M} \mid\} \subseteq \operatorname{Red}_{C}$; which exactly means that $|\mathcal{M}| \subseteq \operatorname{Red}_{B \rightarrow C}$ iff $M \in \operatorname{Red}_{B \rightarrow C}$.

- If $A=A_{1} \times A_{2}$ : then for $i \in\{1,2\},\left(\pi_{i} M\right) \rightarrow\left(\pi_{i} \mathcal{M}\right)$ so that by IH, $\left(\pi_{i} M\right) \in \operatorname{Red}_{A_{i}}$ iff $\left(\pi_{i} \mathcal{M}\right) \subseteq \operatorname{Red}_{A_{i}}$; which exactly means that $|\mathcal{M}| \subseteq \operatorname{Red}_{A_{1} \times A_{2}}$ iff $M \in \operatorname{Red}_{A_{1} \times A_{2}}$.

(2) The reducibility candidates over $\operatorname{Red}_{A}$ are $\Rightarrow$-saturated. This is a trivial induction on $\Rightarrow$ using the $\rightarrow$-saturation for the $(R-+)$ case.

(3) $\operatorname{Red}_{A}$ is inhabited by a value. By induction on $A: \mathbf{0} \in \operatorname{Red}_{\mathrm{NAT}}, \lambda x . V \in \operatorname{Red}_{A \rightarrow B}$ and $\langle U, V\rangle \in \operatorname{Red}_{A \times B}$ whenever $U \in \operatorname{Red}_{A}$ and $V \in \operatorname{Red}_{B}$. 
(4) The reducibility candidates $M$ over $R e d_{A} \Rightarrow$-reduce to $\llbracket M \rrbracket_{a}$. This goes by induction on $A$ :

- Trivial for $A=$ NAT.

- Let $M \in \operatorname{Red}_{B \rightarrow C}$, there is a value $V \in \operatorname{Red}_{B}$, thus $(M V) \in \operatorname{Red}_{C}$ and $M V \Rightarrow \llbracket M V \rrbracket_{a}$ by IH; we can conclude using Lemma 3.4.

- Similar for products.

(5) Every term $x_{1}: A_{1}, \ldots, x_{n}: A_{n} \vdash M: B$ is a candidate in the sense that if $V_{i} \in \operatorname{Red}_{A_{i}}$ then $M\left[V_{1} / x_{1}, \ldots, V_{n} / x_{n}\right] \in \operatorname{Red}_{B}$. By induction on the type derivation. The only difficult cases are applications, recursion, and binary probabilistic choices:

- For the application, we have to show that if $M \in \operatorname{Red}_{A \rightarrow B}$ and $N \in \operatorname{Red}_{A}$ then $(M N) \in \operatorname{Red}_{B}$. But since $N \in \operatorname{Red}_{A}$ we get that $N \Rightarrow \llbracket N \rrbracket_{a}$ with $\left|\llbracket N \rrbracket_{a}\right| \subseteq \operatorname{Red}_{A}$. This means that $\left|M \llbracket N \rrbracket_{a}\right| \subseteq \operatorname{Red}_{B}$ and that $M \llbracket N \rrbracket_{a} \Rightarrow \llbracket M \llbracket N \rrbracket_{a} \rrbracket_{a}$ supported into $\operatorname{Red}_{B}$. We conclude by Lemma 3.5 that $\mathcal{U}=\llbracket M N \rrbracket_{a}$ and thus that $(M N) \in \operatorname{Red}_{B}$.

- For the operator rec, We have to show that if $U \in \operatorname{Red}_{A}$ and $V \in \operatorname{Red}_{\mathrm{NAT} \rightarrow A \rightarrow A}$ then for all $n \in \mathbb{N},(\operatorname{rec}\langle U, V, \boldsymbol{n}\rangle) \in \operatorname{Red}_{A}$. We proceed by induction on $n$ :

- If $n=0: \operatorname{rec}\langle U, V, \mathbf{0}\rangle \rightarrow\{U\} \subseteq \operatorname{Red}_{A}$ and we conclude by saturation.

- Otherwise: $\operatorname{rec}\langle U, V,(\boldsymbol{n}+\mathbf{1})\rangle \rightarrow\{V \boldsymbol{n}(\operatorname{rec}\langle U, V, \boldsymbol{n}\rangle)\} \subseteq \operatorname{Red}_{A}$ since $(\operatorname{rec}\langle U, V, \boldsymbol{n}\rangle) \in$ $\operatorname{Red}_{A}$ by IH and since $\boldsymbol{n} \in \operatorname{Red}_{\mathrm{NAT}}$ and $V \in \operatorname{Red}_{\mathrm{NAT} \rightarrow A \rightarrow A}$, we conclude by saturation.

- For the operator $\oplus$, if $M, N \in \operatorname{Red}_{A}$ then $\left|\left\{\begin{array}{c}M \mapsto \frac{1}{2} \\ N \mapsto \frac{1}{2}\end{array}\right\}\right| \subseteq \operatorname{Red}_{A}$, and, by $\rightarrow$-saturation, $(M \oplus N) \in \operatorname{Red}_{A}$.

The thesis, as usual, can be proved as a corollary of points 4 and 5 .

Notice that this theorem does not apply to $\mathbb{T}^{\mathrm{X}}$ (and a fortiori to $\mathbb{T}^{\oplus, \mathrm{R}, \mathrm{X}}$ ) because step (5) of the proof would not hold.

Theorem 3.7. The accessible evaluation of a term $M \in \mathbb{T}^{\oplus, \mathrm{R}}$ is its evaluation, i.e., $\llbracket M \rrbracket_{a}=$ $\llbracket M \rrbracket$. Moreover, any term $M$ is almost surely terminating.

Proof. By a trivial induction on $\Rightarrow$, we can easily show that if $M \Rightarrow \mathcal{M}$ then $\llbracket M \rrbracket=\llbracket \mathcal{M} \rrbracket$. As a consequence, $\llbracket M \rrbracket=\llbracket \llbracket M \rrbracket_{a} \rrbracket=\llbracket M \rrbracket_{a}$. By another trivial induction on $\Rightarrow$ we can moreover show that if $\mathcal{M} \Rightarrow \mathcal{N}$ then $\|\mathcal{M}\|=\|\mathcal{N}\|$, which yields the thesis.

Corollary 3.8. Any term $M \in \mathbb{T}^{\oplus}$ is positively almost-surely terminating.

Proof. By an induction on $\Rightarrow$ we can show that if $M \Rightarrow \llbracket M \rrbracket$ (resp. $\mathcal{M} \Rightarrow \llbracket \mathcal{M} \rrbracket$ for $\mathcal{M}$ finitely supported) then $M \rightarrow^{*} \llbracket M \rrbracket\left(\right.$ resp. $\left.\mathcal{M} \rightarrow^{*} \llbracket \mathcal{M} \rrbracket\right)$ :

- $(R-0)$ is trivial.

- $(R-+)$ is immediate once we remark that in $\mathbb{T}^{\oplus}$ whenever $M \rightarrow \mathcal{M}$, necessarily $\mathcal{M}$ is finitely supported and we can use our induction hypothesis.

- If for all $M \in|\mathcal{M}|, M \Rightarrow \llbracket M \rrbracket$, then by IH, $M \rightarrow^{n_{M}} \llbracket M \rrbracket$ for some $n_{M} \in \mathbb{N}$. Moreover, since $|\mathcal{M}|$ is finite, we can set $n=\sup _{M \in|\mathcal{M}|}$ so that $M \rightarrow^{\leq n} \llbracket M \rrbracket$ and $\mathcal{M} \rightarrow^{\leq n} \int_{\mathcal{M}} \llbracket M \rrbracket d M=\llbracket \mathcal{M} \rrbracket$. The reduction time of a term is then bounded by $n$ such that $M \rightarrow^{n} \llbracket M \rrbracket$.

Notice that Corollary 3.8 does not apply to $\mathbb{T}^{\mathrm{R}}$ (and a fortiori to $\mathbb{T}^{\oplus, \mathrm{R}, \mathrm{X}}$ ) because the second bullet of the proof would not be verified. 
3.2. Mapping to $\mathbb{T}$. Positive almost sure termination of terms in $\mathbb{T}^{\oplus}$ is not the only consequence of Theorem 3.7. In fact, the finiteness of the resulting distribution over values allows a finite representation of $\mathbb{T}^{\oplus}$-distributions by $\mathbb{T}$-definable functions. Indeed, we can consider an extension of classic system $\mathbb{T}$ with a single memory cell of type NAT that we use to store (the binary encoding of) the outcomes of the coin flips we will perform in the future. If we denote $c$ the memory-cell, this means that the $\oplus$ can be encoded ${ }^{3}$ :

$$
(M \oplus N)^{*} \quad:=\quad \text { if }\left(\bmod _{2} c\right) \text { then }\left(c:=\frac{c}{2} ; M^{*}\right) \text { else }\left(c:=\frac{c}{2} ; N^{*}\right)
$$

From Theorem 3.6, we know that for any $M \in \mathbb{T}^{\oplus}(\mathrm{NAT})$, there is $n \in \mathbb{N}$ such that $M \rightarrow^{n} \llbracket M \rrbracket$. Since the execution is bounded by $n$, there cannot be more than $n$ successive probabilistic choice so that:

$$
\llbracket M \rrbracket=\left\{k \mapsto \frac{\#\left\{m<2^{n} \mid k=\mathbb{N}\left(c:=\boldsymbol{m} ; M^{*}\right)\right\}}{2^{n}}\right\} .
$$

Using a well known state-passing style transformation, we can enforce $\left(c:=\boldsymbol{m} ; M^{*}\right)$ into a term of $\mathbb{T}$. Then, using a simple recursive operation on $m$, we can represent the whole $\#\left\{m<2^{n} \mid k=\mathbb{N}\left(c:=\boldsymbol{m} ; M^{*}\right)\right\}$ into the result of a term $k: \mathbb{N} \vdash N: \mathbb{N}$ so that $\lambda k . N$ define a function that represent the distribution $\llbracket M \rrbracket$.

Example 3.9. Take the term $M=\operatorname{rec}\langle\mathbf{0}, \lambda x y . y \oplus \mathrm{S} y, \mathbf{2}\rangle$ from Example 3.2. Its encoding in $\mathbb{T}$ is

$$
M^{*}=\operatorname{rec}\left\langle\mathbf{0}, \lambda x y \text {. if }(\bmod c \mathbf{2}) \text { then }\left(c:=\frac{c}{2} ; y\right) \text { else }\left(c:=\frac{c}{2} ; \mathrm{S} y\right), \mathbf{2}\right\rangle .
$$

By a standard state passing lifting (and a few simplifications) we obtain the term:

$$
M^{\sim}=\operatorname{rec}\langle\lambda c .(\mathbf{0}, c), \lambda x y c . \text { if }(\bmod c \mathbf{2}) \text { then } y(\operatorname{div} c \mathbf{2}) \text { elses }(y(\operatorname{div} c \mathbf{2})), \mathbf{2}\rangle
$$

As we know that there are at most two choices, we can count the number of $c$ below 4 which result to a certain $u$, getting:

$$
M^{\$}:=\lambda u \cdot \operatorname{rec}\left\langle\mathbf{0}, \lambda x y \text {. if }\left(\pi_{1}\left(M^{\sim} x\right)==u\right) \text { then Sy else } y, \mathbf{4}\right\rangle .
$$

Then we have:

$$
\llbracket M \rrbracket=\left\{k \mapsto \frac{\mathrm{NF}\left(M^{\$} \boldsymbol{k}\right)}{4}\right\} .
$$

What remains to be shown is that this encoding can be made parameteric, in the sense that for any $M \in \mathbb{T}^{\oplus}(\mathrm{NAT} \rightarrow \mathrm{NAT})$, we can generate $M_{\downarrow} \in \mathbb{T}(\mathrm{NAT} \rightarrow \mathrm{NAT} \rightarrow \mathrm{NAT})$ and $M_{\#} \in \mathbb{T}(\mathrm{NAT} \rightarrow \mathrm{NAT})$ such that for all $n \in \mathbb{N}$ :

$$
\llbracket M \boldsymbol{n} \rrbracket=\left\{\boldsymbol{k} \mapsto \frac{\#\left\{m<2^{\mathbb{N F}\left(M_{\#} \boldsymbol{n}\right)} \mid k=\mathbb{F}\left(M_{\downarrow} \boldsymbol{n}\right)\right\}}{2^{\mathbb{N F}\left(M_{\#} \boldsymbol{n}\right)}}\right\} .
$$

The difficulty, here, comes from the bound $M_{\#}$ that have to be computed dynamically by a complex monadic encoding. To this purpose, a translation of $\mathbb{T}^{\oplus}$ into $\mathbb{T}$ has to be appropriately defined.

First of all, let us define two maps $((\cdot))$ and $((\cdot))_{\boldsymbol{V}}$ on types as follows:

$$
\begin{aligned}
((A)) & :=\left(\mathrm{NAT} \rightarrow((A))_{\boldsymbol{V}}\right) \times \mathrm{NAT} ; & & ((\mathrm{NAT}))_{\boldsymbol{V}}:=\mathrm{NAT} ; \\
((A \rightarrow B))_{\boldsymbol{V}}: & :=((A))_{\boldsymbol{V}} \rightarrow((B)) ; & & ((A \times B))_{\boldsymbol{V}}:=((A))_{\boldsymbol{V}} \times((B))_{\boldsymbol{V}} .
\end{aligned}
$$

\footnotetext{
${ }^{3}$ Notice that conditionals, parity and fractions are easily implementable in $\mathbb{T}$.
} 
This can be seen as the monadic lifting of the probabilistic monad. The maps $((\cdot))$ and $((\cdot))_{\boldsymbol{V}}$ can be generalized to type environments in a natural way. Their extension to $\mathbb{T}^{\oplus}$ terms, for $((\cdot))$, and to $\mathbb{T}^{\oplus}$ extended values, for $((\cdot))_{\boldsymbol{V}}$, is the core of the embedding, and will be defined shortly. In the meantime, it is instructive to examine the properties we expect from these maps. First of all, whenever $\Gamma \vdash M: A$ and $\Gamma \vdash V: A$, it holds that

$$
((\Gamma))_{V} \vdash((M)):((A)) ; \quad((\Gamma))_{V} \vdash((V))_{V}:((A))_{V} ;
$$

in $\mathbb{T}$. With a slight abuse of notation, we see the type $((A))$, which by definition is a product type, as given through two components $((A))_{\downarrow}:=\mathrm{NAT} \rightarrow((A))_{V}$ and $((A))_{\#}:=\mathrm{NAT}$. Accordingly, we denote $M_{\downarrow}:=\pi_{1} M:((A))_{\downarrow}$ and $M_{\#}:=\pi_{2} M:((A))_{\#}$ whenever $M:((A))$. Similarly, we may directly define $((M))_{\downarrow}$ and $((M))_{\#}$ whenever $((M))=\left\langle((M))_{\downarrow},((M))_{\#}\right\rangle$.

We can now give a precise (although laborious) definition of the maps above. What is important for the rest of the development is that for every natural number $n,((\boldsymbol{n}))_{\boldsymbol{V}}=\boldsymbol{n}$, and that $((\lambda y \cdot M))_{\boldsymbol{V}}=\lambda y \cdot((M))$. The encoding $((\cdot))_{\boldsymbol{V}}$ of extended values is given by:

$$
\begin{array}{rlrl}
((\mathrm{S}))_{\boldsymbol{V}} & :=\lambda y \cdot((\mathrm{S} y)) ; & ((\mathrm{S} V))_{\boldsymbol{V}}:=\mathrm{S}((V))_{\boldsymbol{V}} ; & ((\mathbf{0}))_{\boldsymbol{V}}:=\mathbf{0} ; \\
\left(((\langle M, N\rangle))_{\boldsymbol{V}}:\right. & :=\left\langle((M))_{\boldsymbol{V}},((N))_{\boldsymbol{V}}\right\rangle ; & ((\lambda x \cdot M))_{\boldsymbol{V}}:=\lambda x \cdot((M)) ; & ((x))_{\boldsymbol{V}}:=x ; \\
\left(\left(\pi_{i}\right)\right)_{\boldsymbol{V}}:=\lambda x \cdot \operatorname{ret}\left(\pi_{i} x\right) ; & & \\
((\operatorname{rec}))_{\boldsymbol{V}}: & :=\lambda\langle u, v, w\rangle \cdot \operatorname{rec}\langle\operatorname{ret} u, \lambda x y \cdot(v x)=\ll y, w\rangle ;
\end{array}
$$

where ret and $=\ll$ are the return and the bind of the underlying $\operatorname{monad}^{4}$ :

$$
\begin{array}{ccc}
\text { ret }:((A))_{V} \rightarrow((A)) ; & \text { ret }:=\lambda x .\langle\lambda y \cdot x, \mathbf{0}\rangle ; \\
(\cdot)=\ll(\cdot):((A \rightarrow B)) \times((A)) \rightarrow((B)) ; & M=\ll N:=\left\langle M=\ll_{\downarrow} N, M=\ll_{\#} N\right\rangle .
\end{array}
$$

However, the computation of the bind is complex. Intuitively, the right part is computing the number of choices in $M$ in $N$ and in all the possibles outcomes $(U V)$ for $U \in|\llbracket M \rrbracket|$ and $V \in|\llbracket N \rrbracket|$. Of course we take the least upper bound of those outcomes as they are independent:

$$
\begin{aligned}
& (\cdot)=\ll_{\#}(\cdot):((A \rightarrow B)) \times((A)) \rightarrow \mathrm{NAT} ; \\
& M=\ll_{\#} N:=M_{\#}+N_{\#}+\max _{x<2^{M_{\#}}} \max _{y<2^{N_{\#}}}\left(M_{\downarrow} x\left(N_{\downarrow} y\right)\right)_{\#} ;
\end{aligned}
$$

where we use the following syntactical sugar:

$$
\begin{array}{rlrl}
(\cdot)+(\cdot): \mathrm{NAT} \times \mathrm{NAT} \rightarrow \mathrm{NAT} ; & M+N & :=\operatorname{rec}\left\langle M, \lambda \_x . \mathrm{S} x, N\right\rangle ; \\
2^{(\cdot)}: \mathrm{NAT} \rightarrow \mathrm{NAT} ; & 2^{M} & :=\operatorname{rec}\left\langle\mathbf{1}, \lambda \_y . \operatorname{rec}\left\langle y, \lambda \_v . \mathrm{S} v, y\right\rangle, M\right\rangle ; \\
\max (N) & :=\operatorname{rec}\left\langle\mathbf{0}, \lambda \_y . N \vee y, M\right\rangle ; \\
(\cdot) \vee(\cdot): \mathrm{NAT} \times \mathrm{NAT} \rightarrow \mathrm{NAT} ; & M \vee N & :=\operatorname{rec}\left\langle\lambda u . u, \lambda x y u . \mathrm{S}\left(\operatorname{rec}\left\langle x, \lambda a_{-} . y a, u\right\rangle\right), M\right\rangle N .
\end{array}
$$

The first member of the bind is also complex. It is taking a stream of probabilistic choices $s$, computes $M$ over this choices, then shifts the stream $s$ in order to remove the choices relative to $M$, this way we can compute the result from $N$. After all this, we obtain an object of type $((B))$, but our job is not finished yet: we have to select the first member, to

\footnotetext{
${ }^{4}$ Technically, this bind is not the usual bind, but a lifted version.
} 
which we give what remains of the stream (computed by shifting $x$ twice).

$$
\begin{aligned}
& (\cdot)=\ll_{\downarrow}(\cdot):((A \rightarrow B)) \times((A)) \rightarrow \mathrm{NAT} \rightarrow((B))_{V} ; \\
& M=\ll_{\downarrow} N:=\lambda s .\left(M_{\downarrow} s\left(N_{\downarrow}\left(\operatorname{shift} s M_{\#}\right)\right)\right)_{\downarrow}\left(\text { shift } s\left(M_{\#}+N_{\#}\right)\right) ;
\end{aligned}
$$

where we use the following syntactical sugar:

$$
\begin{aligned}
\text { shift }: N A T & \rightarrow \mathrm{NAT} \rightarrow \mathrm{NAT} ; \quad \text { shift }:=\lambda s y \cdot \operatorname{rec}\left\langle s, \lambda u . d i v_{2}, y\right\rangle ; \\
\operatorname{div}_{2}: \mathrm{NAT} & \rightarrow \mathrm{NAT} ; \quad \operatorname{div}_{2}:=\lambda x \cdot \operatorname{rec}\left\langle\lambda_{-} . \mathbf{0}, \lambda_{-} v w . \operatorname{ite}\langle w, \mathrm{~S}(v \mathbf{0}), v \mathbf{1}\rangle, x\right\rangle \mathbf{0} .
\end{aligned}
$$

The encoding $((\cdot))$ is given through the return and the bind operations:

$$
((V)):=\operatorname{ret}((V))_{V} ; \quad((M N)):=((M))=\ll((N)) .
$$

The binary choice is the defined as expected:

$$
\begin{aligned}
((M \oplus N))_{\#} & :=((M))_{\#} \vee((N))_{\#} ; \\
((M \oplus N))_{\downarrow} & :=\lambda x . \operatorname{ite}\left\langle\bmod _{2} x,((M))_{\downarrow}\left(\operatorname{div}_{2} x\right),((N))_{\downarrow}\left(\operatorname{div}_{2} x\right)\right\rangle ;
\end{aligned}
$$

where we use the following syntactical sugar:

$$
\begin{array}{rlrl}
\text { ite } & \text { NAT } \times A \times A \rightarrow A ; & \text { ite } & :=\lambda x \cdot \operatorname{rec}\left\langle\pi_{3} x, \lambda_{--} \cdot \pi_{2} x, \pi_{1} x\right\rangle ; \\
\bmod _{2}: \text { NAT } & \rightarrow \text { NAT } ; & \bmod _{2}:=\lambda x \cdot \operatorname{rec}\left\langle\mathbf{0}, \lambda \_v . \text { ite }\langle v, \mathbf{0}, \mathbf{1}\rangle, x\right\rangle .
\end{array}
$$

The byproduct of this relatively complex encoding is the fact that whatever distribution one is able to compute in $\mathbb{T}^{\oplus}$ can also be computed back in $\mathbb{T}$, and that this scales to first-order functions:

Theorem 3.10. Distributions in $\mathbb{T}^{\oplus}$ are finitely parameterically representable by $\mathbb{T}$-definable functions, i.e. for any $M: \mathrm{NAT} \rightarrow \mathrm{NAT}$ in $\mathbb{T}^{\oplus}$ there are $F: \mathrm{NAT} \rightarrow \mathrm{NAT} \rightarrow \mathrm{BIN}$ and $Q: \mathrm{NAT} \rightarrow \mathrm{NAT}$ in $\mathbb{T}$ such that for all $n$ :

$$
\llbracket M n \rrbracket=\{\boldsymbol{k} \mapsto \mathbb{F}(F \boldsymbol{n} \boldsymbol{k})\} ; \quad \forall k \geq \mathbb{N}(Q \boldsymbol{n}) . \mathbb{N}(F \boldsymbol{n} \boldsymbol{k})=\mathbf{0} .
$$

\section{Countable Probabilistic Choice}

4.1. Multistep Semantics. We have seen that none of Theorem 3.6, Theorem 3.7 and Corollay 3.8 hold in $\mathbb{T}^{\mathrm{X}}$. Indeed Theorem 3.6, which is a prologue to the other two, does not hold on terms like, e.g., $\mathrm{X}\langle\mathbf{0}, \mathrm{S}\rangle$ that will never $\Rightarrow$-reduce to a value distribution. The fragment $\mathbb{T}^{\mathrm{R}}$ is more interesting, as both Theorem 3.6 and Theorem 3.7 hold. However, as we have seen in Theorem 2.15, positive almost sure normalization (and Theorem 3.8) do not hold. This is because we are manipulating infinitely supported distributions (due to the reduction rule of $R$ ).

Example 4.1. Recall that $\mathrm{X}_{\mathrm{R}}:=\lambda x \cdot \operatorname{rec}\left\langle\pi_{2} x, \lambda z \cdot \pi_{1} x, \mathrm{R}\right\rangle$ is the encoding of $\mathrm{X}$ into $\mathbb{T}^{\mathrm{R}}$. We have $\mathrm{X}: \mathrm{R}\langle\mathrm{SS}, \mathbf{0}\rangle \Rightarrow\left\{\mathbf{2} \boldsymbol{n} \mapsto \frac{1}{2^{n+1}} \mid n \geq 0\right\}$; indeed, if we fix $U_{n}=\operatorname{rec}\left\langle\pi_{2}\langle\mathrm{SS}, \mathbf{0}\rangle, \lambda z . \pi_{1}\langle\mathrm{SS}, \mathbf{0}\rangle, \boldsymbol{n}\right\rangle$ : 


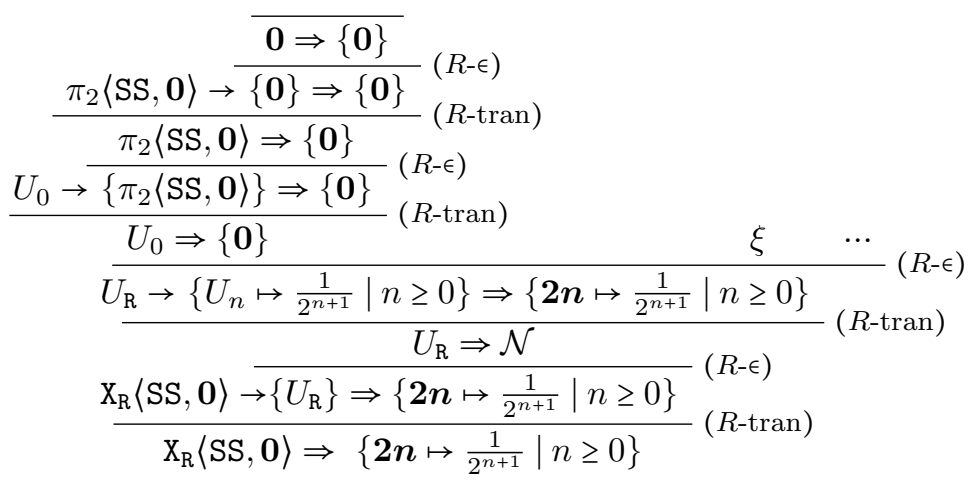

where $\xi$ is the following derivation:

$$
\begin{aligned}
& \frac{\pi_{1}\langle\mathrm{SS}, \mathbf{0}\rangle \mathbf{0} \rightarrow \frac{\overline{\mathrm{SS} \mathbf{0} \Rightarrow\{\mathbf{2}\}}}{\{\mathrm{SS} \mathbf{0}\} \Rightarrow\{\mathbf{2}\}}}{\pi_{1}\langle\mathrm{SS}, \mathbf{0}\rangle \mathbf{0} \Rightarrow\{\mathbf{2}\}}(R \text { - } \epsilon) \\
& \frac{\pi_{1}\langle\mathrm{SS}, \mathbf{0}\rangle\left(\pi_{2}\langle\mathrm{SS}, \mathbf{0}\rangle\right) \rightarrow \frac{\pi_{1}\langle\mathrm{SS}, \mathbf{0}\rangle \mathbf{0} \Rightarrow\{\mathbf{2}\}}{\left\{\pi_{1}\langle\mathrm{SS}, \mathbf{0}\rangle \mathbf{0}\right\} \Rightarrow\{\mathbf{2}\}}(R-\epsilon)}{\pi_{1}\langle\mathrm{SS}, \mathbf{0}\rangle\left(\pi_{2}\langle\mathrm{SS}, \mathbf{0}\rangle\right) \Rightarrow\{\mathbf{2}\}}(R \text {-tran })
\end{aligned}
$$

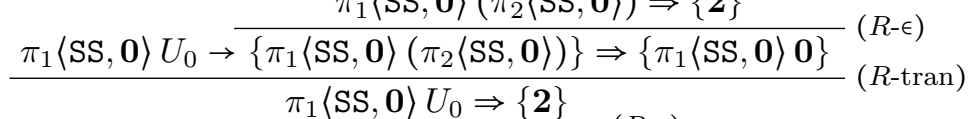

$$
\begin{aligned}
& \frac{U_{1} \rightarrow \frac{\left.\pi_{1}\langle\mathrm{SS}, \mathbf{0}\rangle U_{0}\langle\mathrm{SS}, \mathbf{0}\rangle U_{0}\right\} \Rightarrow\{\mathbf{2}\}}{U_{1} \Rightarrow\{\mathbf{2}\}}(R-\epsilon)}{(R \text {-tran })}
\end{aligned}
$$

we can see that the tree is infinite due to the second application of $(R-\epsilon)$; but, despite being infinite, and with an infinite height, each subtree above the second application of $(R-\epsilon)$ is finite, making the derivation correct. However, $\mathrm{X}\langle\mathrm{SS}, \mathbf{0}\rangle \not\left\{\mathbf{2} \boldsymbol{n} \frac{1}{2^{n+1}} \mid n \geq 0\right\}$. We can approach this distribution, but it is impossible to $\Rightarrow$-reduce to a value distribution.

Remember that $\mathbb{T}^{\mathrm{R}}$ and $\mathbb{T}^{\mathrm{X}}$ are equivalent, so why such a difference? This is due to the discrepancy in nature between their execution trees. Indeed, we have seen that the execution trees are finitely branching in $\mathbb{T}^{\mathrm{X}}$, but with infinite paths, while those of $\mathbb{T}^{\mathrm{R}}$ are infinitely branching, but with finite paths. Since multistep reduction somehow reflects those execution trees, we can see that we only need derivations with infinite arity to get a correct multistep semantics for $\mathbb{T}^{R}$. The whole point is that we can perform transfinite structural induction over these trees. Indeed, by considering the reduction trees themselves with the inclusion (or subtree) order gives you a well-founded poset, recalling that there is no infinite path. If one want to unfold this well-founded poset into an ordinal, then it should be the smallest ordinal $o$ such that $o=1+\omega o$, i.e., $o=\omega^{\omega}$. This is unusual in operational semantics, where finitary induction suffices in most cases. Remark that, due to the encoding of $\oplus$ and $\mathrm{X}$ into $\mathbb{T}^{\mathrm{R}}$, Theorem 3.7 subsumes Theorem 2.13. Remark, moreover, that we did not have to go through the definition of approximants. Nonetheless, those approximations exists and point out that $\mathbb{T}^{\oplus}$ should be approximating $\mathbb{T}^{R}$ in some way or another. This is precisely what we are going to do in the next section.

4.2. The Approximants: State-Bounded Random Integers. In this section, we show that $\mathbb{T}^{\oplus}$ approximates $\mathbb{T}^{\mathrm{R}}$ : for any term $M \in \mathbb{T}^{\mathrm{R}}(\mathrm{NAT})$, there is a term $N \in \mathbb{T}^{\oplus}(\mathrm{NAT} \rightarrow \mathrm{NAT})$ that represents a sequence approximating $M$ uniformly. We will here make strong use of 


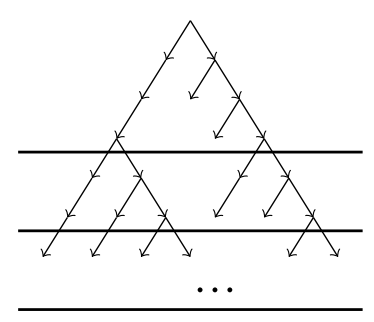

(a) Horizontal

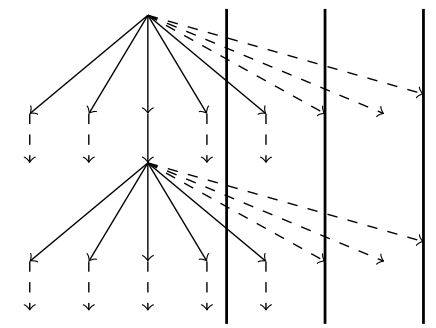

(b) Vertical

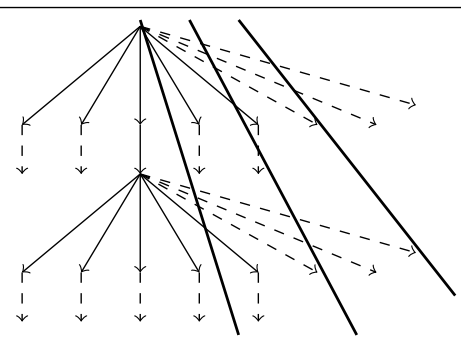

(c) Biaised

FiguRE 5. Various Forms of Approximation

the fact that $M$ has type NAT. This is a natural drawback when we understand that the encoding $(\cdot)^{\dagger}$ on which the result above is based is not direct, but goes through another state-passing transformation.

A naive idea would be to use $\mathbb{T}^{\oplus}$ and to stop the evaluation after a given reduction time as schematized in Figure 5a. Despite the encoding to be a nightmare, this should be encodable in $\mathbb{T}^{\oplus}$. However, for the convergence time to be independent from the term and uniform, there is virtually no hope. That is why we have switched to $\mathbb{T}^{\mathrm{R}}$, which carries much nicer properties as seen in the previous chapter. The basic idea behind the embedding $(\cdot)^{\dagger}$ is to mimic any instance of the R operator in the source term by some term $\mathbf{0} \oplus(\mathbf{1} \oplus(\cdots(\boldsymbol{n} \oplus \perp) \cdots)$, where $n$ is sufficiently large, and $\perp$ is an arbitrary value of type NAT. Of course, the semantics of this term is not the same as that of $\mathrm{R}$, due to the presence of $\perp$; however, $n$ will be chosen sufficiently large for the difference to be negligible. Notice, moreover, that this term can be generalized into the following parametric form $\mathrm{R}^{\ddagger}:=\lambda n . \operatorname{rec}\langle\perp,(\lambda x . \mathrm{S} \oplus(\lambda y . \mathbf{0}))\rangle n$.

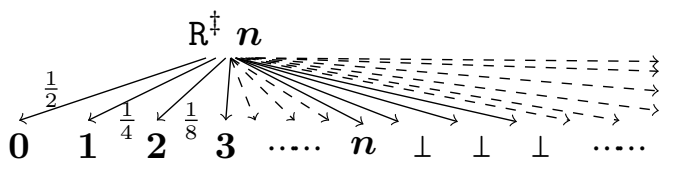

Once $\mathrm{R}^{\ddagger}$ is available, a natural candidate for the encoding $(\cdot)^{\dagger}$ would be to consider something like $M^{\ddagger}:=\lambda z \cdot M\left[\left(\mathrm{R}^{\ddagger} z\right) / \mathrm{R}\right]$. In the underlying execution tree, $\left(M^{\ddagger} \boldsymbol{n}\right)$ correctly simulates the first $n$ branches of each $\mathrm{R}$ (which had infinite-arity), but truncates the rest with garbage terms $\perp$. As schematized in Figure 5b, by increasing $n$, we can hope to obtain the $M$ at the limit.

The question is whether the remaining non-truncated tree has a "sufficient weight", i.e., that there is a minimal bound to the probability to stay in this non-truncated tree. However, in general $(\cdot)^{\ddagger}$ fails on this point, not achieving to approximate $M$ uniformly. In fact, this probability is basically $\left(1-\frac{1}{2^{n}}\right)^{d}$ where $d$ is its depth. Since in general the depth of the non-truncated tree can grow very rapidly with respect to $n$ in a powerful system like $\mathbb{T}$, there is no hope for this transformation to perform a uniform approximation. It might well be possible to perform a complex monadic transformation in the style of Section 3.2, that computes a function relating the size $n$ to the depth $d$ of the execution tree. But there is a much easier solution.

The solution we are using is to have the precision $m$ of $\mathbf{0} \oplus(\mathbf{1} \oplus(\cdots(\boldsymbol{m} \oplus \perp) \cdots))$ to dynamically grow along the computation, as schematized in Figure 5c. More specifically, 


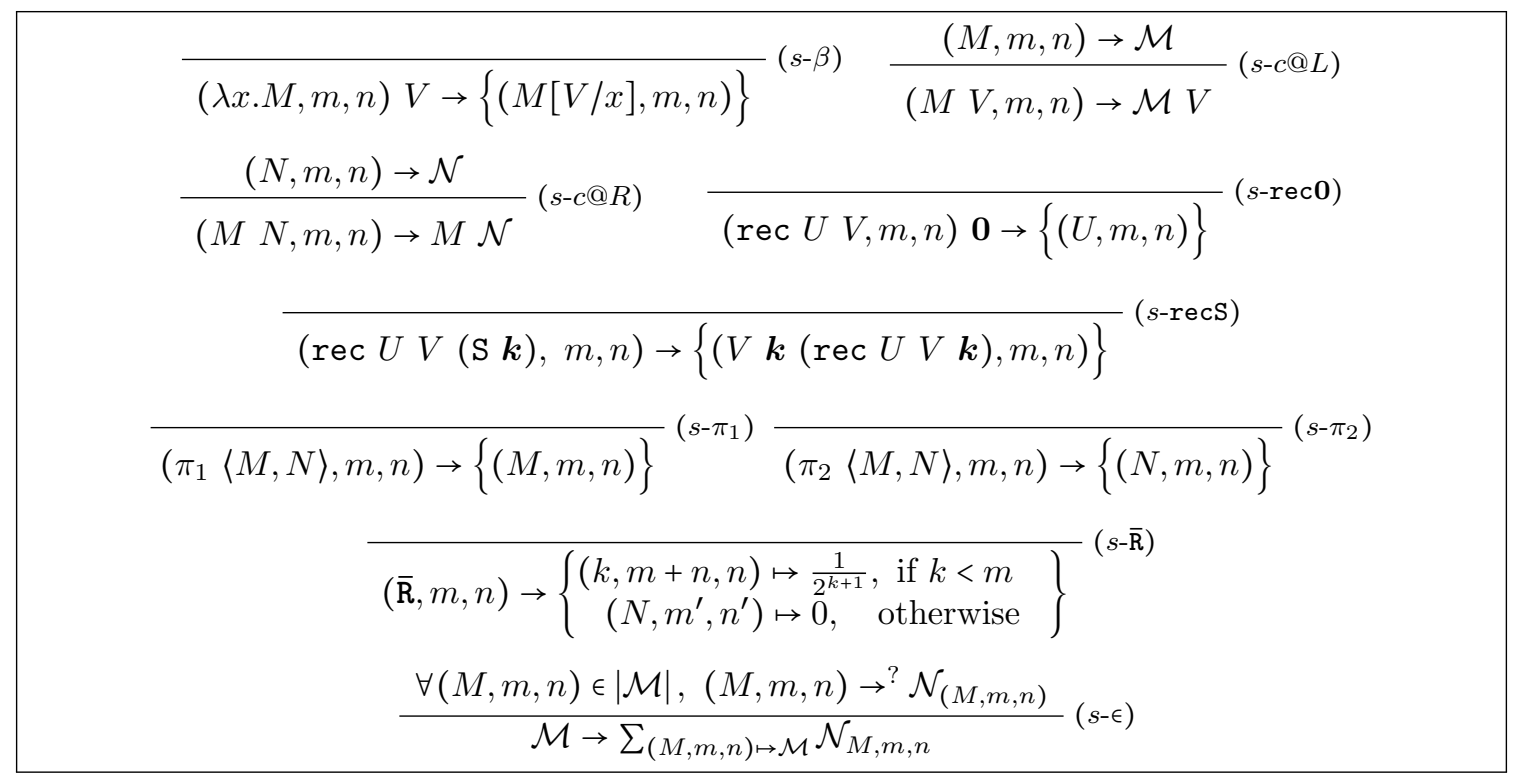

Figure 6 . Operational semantics of $\mathbb{T}^{\bar{R}}$

in the approximants $M^{\dagger} \boldsymbol{n}$, the growing speed of $m$ will increase with $n$ : in the $n$-th approximation $M^{\dagger} \boldsymbol{n}$, R will be simulated as $\mathbf{0} \oplus(1 \oplus(\cdots(\boldsymbol{m} \oplus \perp) \cdots))$ and, somehow, $m$ will be updated to $m+n$. Why does it work? Simply because even for an (hypothetical) infinite and complete execution tree of $M$, we would stay inside the $n^{\text {th }}$ non-truncated tree with probability $\prod_{k \geq 0}\left(1-\frac{1}{2^{k * n}}\right)$ which is asymptotically above $\left(1-\frac{1}{n}\right)$.

Implementing this scheme in $\mathbb{T}^{\oplus}$ requires a feature which is not available (but which can be encoded), namely ground-type references. We then prefer to show that the just described scheme can be realized in an intermediate language called $\mathbb{T}^{\overline{\mathrm{R}}}$, whose operational semantics is formulated not on terms, but rather on triples in the form $(M, m, n)$, where $M$ is the term currently being evaluated, $m$ is the current approximation threshold value, and $n$ is the value of which $m$ is incremented whenever $\mathrm{R}$ is simulated. The operational semantics is standard, except for the following rule:

$$
\overline{(\overline{\mathrm{R}}, m, n) \rightarrow\left\{(k, m+n, n) \mapsto \frac{1}{2^{k+1}} \mid k<m\right\}}(r-\overline{\mathrm{R}})
$$

Notice how this operator behaves similarly to $R$ with the exception that it fails when drawing too big of a number (i.e., bigger that the fist state $m$ ). Notice that the failure is represented by the fact that the resulting distribution does not necessarily sum to 1 . The intermediate language $\mathbb{T}^{\overline{\mathrm{R}}}$ is able to approximate $\mathbb{T}^{\mathrm{R}}$ at every order (Theorem 4.6 below). Moreover, the two memory cells can be shown to be expressible in $\mathbb{T}^{\oplus}$, again by way of a continuation-passing transformation. Crucially, the initial value of $n$ can be passed as an argument to the encoded term.

Definition 4.2. For any $M \in \mathbb{T}^{\mathrm{R}}$, the expression $M^{*}$ stands for $M[\overline{\mathrm{R}} / \mathrm{R}]$. We say that $(M, m, n) \in$ $\mathbb{T}^{\overline{\mathrm{R}}}$ if $m, n \in \mathbb{N}$ and $M=N^{*}$ for some $N \in \mathbb{T}^{\mathrm{R}}$. Similarly, $\mathfrak{D}\left(\mathbb{T}^{\overline{\mathrm{R}}}\right)$ is the set of probabilistic distributions over $\mathbb{T}_{C}^{\bar{R}} \times \mathbb{N}^{2}$, i.e., over the terms plus states. The reduction rules of system $\mathbb{T}$ with state-bounded random integers are given by Figure 6 . 
For any $m$ and $n$, the behavior of $M$ and $\left(M^{*}, m, n\right)$ are similar, except that $\left(M^{*}, m, n\right)$ will "fail" more often. In other words, any $\left(M^{*}, m, n\right)$ approximates the behavior of $M$ from below:

Lemma 4.3. For any $M \in \mathbb{T}^{\mathrm{R}}$ and any $m, n \in \mathbb{N}$, $\llbracket \rrbracket \geq \llbracket M^{*}, m, n \rrbracket$, i.e., for every $V \in \mathbb{T}_{V}^{\mathrm{R}}$, we have

$$
\llbracket M \rrbracket(V) \geq \sum_{l, p} \llbracket M^{*}, m, n \rrbracket\left(V^{*}, l, p\right) .
$$

Proof. By an easy induction, one can show that for any $\mathcal{M} \in \mathfrak{D}\left(\mathbb{T}^{\mathrm{R}}\right)$ and $\mathcal{N} \in \mathfrak{D}\left(\mathbb{T}^{\overline{\mathrm{R}}}\right)$ if $\mathcal{M} \geq \mathcal{N}, \mathcal{M} \rightarrow \mathcal{L}$ and $\mathcal{N} \rightarrow \mathcal{P}$, then $\mathcal{L} \geq \mathcal{P}$. This ordering is then preserved at the limit so that we get our result.

In fact, the probability of "failure" of any $(M, m, n)_{m, n \in \mathbb{N}}$ can be upper-bounded explicitly. More precisely, we can find an infinite product underapproximating the success rate of $(M, m, n)$ by reasoning inductively over $(M, m, n) \Rightarrow \llbracket(M, m, n) \rrbracket$.

Lemma 4.4. For any $M \in \mathbb{T}^{\overline{\mathrm{R}}}$ and any $m, n \geq 1$

$$
\operatorname{Succ}(M, m, n) \geq \prod_{k \geq 0}\left(1-\frac{1}{2^{m+k n}}\right) .
$$

Proof. We use the following notation:

$$
\#(m, n):=\prod_{k \geq 0}\left(1-\frac{1}{2^{m+k n}}\right) ; \quad \# \mathcal{M}:=\sum_{(M, m, n) \mapsto \mathcal{M}} \#(m, n) .
$$

By induction on $\Rightarrow$, we can show that if $(M, m, n) \Rightarrow \mathcal{M}$ then $\# \mathcal{M}=\#(m, n)$ and that if $\mathcal{N} \Rightarrow \mathcal{M}$ then $\# \mathcal{M}=\# \mathcal{N}:$

- If $(M, m, n) \rightarrow \mathcal{N} \Rightarrow \mathcal{M}$ then $\mathcal{N}$ is either of the form $\{(N, m, n)\}$ or $\left\{\left(N_{i}, m+n, n\right) \mapsto\right.$ $\left.\frac{1}{2^{i+1}} \mid i<m\right\}$ for some $N$ of $\left(N_{i}\right)_{i \leq m}$. In the first case it is clear that $\# \mathcal{N}=\#(m, n)$, but the equality holds also in the second:

$$
\# \mathcal{N}=\sum_{i \leq m} \frac{1}{2^{i+1}} \#(m+n, n)=\left(1-\frac{1}{2^{m}}\right) \#(m+n, n)=\#(m, n) .
$$

By IH, we conclude that $\# \mathcal{M}=\# \mathcal{N}=\#(m, n)$.

- The other cases are immediate.

In particular, we have that:

$$
\begin{array}{rlrl}
\operatorname{Succ}(M, m, n) & =\sum_{(M, m, n) \mapsto \llbracket M, m, n \rrbracket} 1 & \\
& \geq \# \llbracket M \rrbracket & & \text { since } \forall m, n, 1 \geq \#(m, n) \\
& =\#(m, n) & & \text { since }(M, m, n) \Rightarrow \llbracket M, m, n \rrbracket .
\end{array}
$$

This gives us an analytic lower bound to the success rate of $(M, m, n)$. However, it is not obvious that this infinite product is an interesting bound: it is not even clear that it can be different from 0 . This is why we will further underapproximate this infinite product to get a simpler expression whenever $m=n$ :

Lemma 4.5. For any $M \in \mathbb{T}^{\overline{\mathrm{R}}}$ and any $n \geq 4$, we have that

$$
\operatorname{Succ}(M, n, n) \geq 1-\frac{1}{n} .
$$


Proof. By Lemma 4.4 we have that $\operatorname{Succ}(M, n, n) \geq \prod_{k \geq 1}\left(1-\frac{1}{2^{k * n}}\right)$ which is above the product $\prod_{k \geq 1}\left(1-\frac{1}{n^{2} k^{2}}\right)$ whenever $n \geq 4$. This infinite product has been shown by Euler to be equal to $\frac{\sin \left(\frac{\pi}{n}\right)}{\frac{\pi}{n}}$. By an easy numerical analysis we then obtain that $\frac{\sin \left(\frac{\pi}{n}\right)}{\frac{\pi}{n}} \geq 1-\frac{1}{n}$.

This lemma can be restated by saying that the probability of "failure" of $\left(M^{*}, n, n\right)$, i.e. the difference between $\llbracket M^{*}, n, n \rrbracket$ and $\llbracket M \rrbracket$, is bounded by $\frac{1}{n}$. With this we then get our first theorem, which is the uniform approximation of elements of $\mathbb{T}^{\mathrm{R}}$ by those of $\mathbb{T}^{\overline{\mathrm{R}}}$ :

Theorem 4.6. For any $M \in \mathbb{T}^{\mathrm{R}}$ and any $n \in \mathbb{N}$,

$$
\sum_{V}\left|\llbracket M \rrbracket(V)-\Sigma_{l, p} \llbracket M^{*}, n, n \rrbracket\left(V^{*}, l, p\right)\right| \leq \frac{1}{n} .
$$

Proof. By Lemma 4.3, for each $V$ the difference is positive, thus we can remove the absolute value and distribute the sum. We conclude by using the fact that $\operatorname{Succ}(M)=1$ and $\operatorname{Succ}\left(M^{*}, n, n\right) \geq 1-\frac{1}{n}$.

The second theorem, i.e., the uniform approximation of ground elements of $\mathbb{T}^{\mathrm{R}}$ by those of $\mathbb{T}^{\oplus}$, follows immediately:

Theorem 4.7. Distributions in $\mathbb{T}^{\mathrm{R}}(\mathrm{NAT})$ can be approximated by $\mathbb{T}^{\oplus}$-distributions (which are finitely $\mathbb{T}$-representable), i.e., for any $M \in \mathbb{T}^{\mathrm{R}}(\mathrm{NAT})$, there is $M^{\dagger} \in \mathbb{T}^{\oplus}(\mathrm{NAT})$ such that for every natural number $n$, it holds that

$$
\sum_{k}\left|\llbracket M \rrbracket(\boldsymbol{k})-\llbracket M^{\dagger} \boldsymbol{n} \rrbracket(\boldsymbol{k})\right| \leq \frac{1}{n} .
$$

Moreover:

- the encoding is parametric, i.e., for all $M \in \mathbb{T}^{\mathrm{R}}(\mathrm{NAT} \rightarrow \mathrm{NAT})$, there is $M^{\dagger} \in \mathbb{T}^{\oplus}(\mathrm{NAT} \rightarrow \mathrm{NAT})$ such that $(M \boldsymbol{n})^{\dagger}=M^{\dagger} \boldsymbol{n}$ for all $n \in \mathbb{N}$;

- the encoding is such that $\llbracket M \rrbracket(\boldsymbol{k}) \leq \llbracket M^{\dagger} \boldsymbol{n} \rrbracket(\boldsymbol{k})$ only when $k=0$.

Proof. It is clear that in an extension of $\mathbb{T}^{\oplus}$ with two global memory cells $m, n$ and with exceptions, the $\bar{R}$ operator can be encoded by

$$
\overline{\mathrm{R}}:=\operatorname{rec}\langle\lambda u . \perp, \lambda x y u . \mathbf{0} \oplus \mathrm{S}(y u), m:=! m+! n\rangle \mathbf{0},
$$

where $\perp$ is raising an error/exception and $m:=! m+! n$ is returning the value of $m$ before changing the memory cell to $m+n$. Remark that the only objective of the dummy abstraction over $u$ and of the dummy application to $\mathbf{0}$, preventing $\perp$ from being evaluated. We can conclude by referring to the usual state passing style encoding of exceptions and state-monads into $\mathbb{T}$ (and thus into $\mathbb{T}^{\oplus}$ ). In fact, we do not have any requirement over $\perp$, i.e., we can replace $\perp$ by any value $\perp_{A}$ of the correct type $A$ (which is possible since every type is inhabited). In other words, we do not need to implement the exception monad, but only the state monad which we can present easily here:

$$
\begin{aligned}
((A)) & :=\mathrm{NAT}^{3} \rightarrow\left(((A))_{\boldsymbol{V}} \times \mathrm{NAT}^{3}\right) ; & ((\mathrm{NAT}))_{\boldsymbol{V}}:=\mathrm{NAT} ; \\
((A \rightarrow B))_{\boldsymbol{V}}:=((A))_{\boldsymbol{V}} \rightarrow((B)) ; & & ((A \times B))_{\boldsymbol{V}}:=((A)) \times((B)) .
\end{aligned}
$$

The state here is an element of $\mathrm{NAT}^{3}$, the first natural number monitoring the presence of an error along the reduction, the second represents the state $m$ and the third represents 
the state $n$. The encoding $((-))_{V}$ of extended values is the same as for the encoding of Section 3.2:

$$
\begin{array}{rlrl}
((\mathrm{S}))_{\boldsymbol{V}} & :=\lambda y \cdot((\mathrm{S} y))_{\boldsymbol{V}} ; & ((\mathrm{S} V))_{\boldsymbol{V}}:=\mathrm{S}((V))_{\boldsymbol{V}} ; & ((\mathbf{0}))_{\boldsymbol{V}}:=\mathbf{0} ; \\
((\langle M, N\rangle))_{\boldsymbol{V}}: & :=\left\langle((M))_{\boldsymbol{V}},((N))_{\boldsymbol{V}}\right\rangle ; & ((\lambda x \cdot M))_{\boldsymbol{V}}:=\lambda x \cdot((M)) ; & ((x))_{\boldsymbol{V}}:=x ; \\
\left(\left(\pi_{i}\right)\right)_{\boldsymbol{V}}: & :=\lambda x \cdot \operatorname{ret}\left(\pi_{i} x\right) ; & & \\
((\mathrm{rec}))_{\boldsymbol{V}} & :=\lambda\langle u, v, w\rangle \cdot \operatorname{rec}\langle\operatorname{ret} u, \lambda x y \cdot(v x)=\ll y, w\rangle .
\end{array}
$$

Where ret and $=\ll$ are the return and the bind of the considered monad:

$$
\begin{array}{ccc}
\operatorname{ret}:((A))_{V} \rightarrow((A)) ; & \operatorname{ret}:=\lambda x s .\langle x, s\rangle ; \\
(\cdot)=\ll(\cdot):((A \rightarrow B)) \times((A)) \rightarrow((B)) ; & M=\ll N:=\lambda s .(\lambda\langle x, t\rangle \cdot(\lambda\langle y, u\rangle . x y u)(N t))(M s) .
\end{array}
$$

What $M=\ll N$ does is looking at the current state $s$, evaluating $M$ under the state $s$ which results to $\langle x, t\rangle$, then evaluating $N$ under the state $s_{2}$ which results to $\langle y, u\rangle$, and, finally, evaluating $(x y):((B))$ under the state $u$. The encoding $((\cdot))$ is given by the return and the bind operations as well as the encoding of effectful operations:

$$
\begin{aligned}
((V)) & :=\operatorname{ret}((V))_{V} ; & ((M N)) & :=((M))=\ll((N)) ; \\
((M \oplus N)) & :=\lambda s .(((M)) s) \oplus(((N)) s) ; & ((m:=! m+! n)) & :=\lambda\langle e, m, n\rangle \cdot\langle m,\langle e, m+n, n\rangle\rangle ; \\
((\perp)) & :=\lambda\langle e, m, n\rangle \cdot\langle *,\langle\mathbf{1},\langle m, n\rangle\rangle\rangle . & &
\end{aligned}
$$

where $*$ is any term of correct type. In the end, we set

$$
M^{\dagger}:=\lambda x .(\lambda\langle y, e, m, n\rangle . \operatorname{rec} y(\lambda u v .0) e)(((M))\langle x, x\rangle)
$$

for $M \in \mathbb{T}^{\mathrm{R}}(\mathrm{NAT})$. The parametrization is obtained by using the equality $((\boldsymbol{n}))_{\boldsymbol{V}}=\boldsymbol{n}$.

Corollary 4.8. Distributions in $\mathbb{T}^{\mathrm{R}}$ are functionally parametrically representable by $\mathbb{T}$ definable functions, i.e. for any $M: \mathrm{NAT} \rightarrow \mathrm{NAT}$ in $\mathbb{T}^{\mathrm{R}}$ there is $F: \mathrm{NAT} \rightarrow \mathrm{NAT} \rightarrow \mathrm{NAT} \rightarrow \mathrm{BIN}$ and $Q: \mathrm{NAT} \rightarrow \mathrm{NAT} \rightarrow \mathrm{NAT}$ in $\mathbb{T}$ such that for all $m$ and $n:$

$$
\sum_{k \in \mathbb{N}}|\llbracket M \boldsymbol{m} \rrbracket(\boldsymbol{k})-\mathbb{N}(F \boldsymbol{m} \boldsymbol{n} \boldsymbol{k})| \leq \frac{1}{n} ; \quad \forall k \geq \mathbb{N}(Q \boldsymbol{m} \boldsymbol{n}) . \mathbb{N}(F \boldsymbol{m} \boldsymbol{n} \boldsymbol{k})=\mathbf{0} .
$$

\section{SUBRECURSION}

Up to now, $\mathbb{T}^{\oplus, R, X}$ and its fragments' expressiveness has been evaluated by considering programs of type NAT $\rightarrow$ NAT as representing functions from $\mathbb{N}$ to $\mathfrak{D}(\mathbb{N})$. Probabilistic computational models, however, are often treated as representing ordinary functions, like in probabilistic complexity theory [AB09, Pap94], where BPP or ZPP are classes of decision problems. If one wishes to define $\mathbb{T}^{\oplus}$-definable or $\mathbb{T}^{\mathrm{R}}$-definable functions as a set of ordinary functions (say from $\mathbb{N}$ to $\mathbb{N}$ ), it is necessary to somehow collapse the probabilistic output into a deterministic one. As already acknowledged by the complexity community, there are at least two reasonable ways to do so: by using a either Monte Carlo (like in BPP) or Las Vegas observations (like in ZPP).

We recall that a function $f: \mathbb{N} \rightarrow \mathbb{N}$ is $\mathbb{T}$-definable if there is a $\mathbb{T}$ program $\vdash M:$ NAT $\rightarrow$ NAT in $\mathbb{T}$ such that $(M \boldsymbol{n}) \rightarrow^{*} \boldsymbol{f}(\boldsymbol{n})$ for all $n$. We denote the set of $\mathbb{T}$-definable functions as DT. 
5.1. Monte Carlo Observations. In Monte Carlo observations, what one observes is the output with the highest probability, which must be sufficiently high to rule out any ambiguity. The class of Monte Carlo representable functions on $\mathbb{T}^{\oplus}$ (respectively, $\mathbb{T}^{\mathrm{R}}$ ) is the class $\mathbf{B P T} \mathbf{T}^{\oplus}$ (respectively, BPT ${ }^{\mathrm{R}}$ ) of functions $f$ definable by a $\mathbb{T}^{\oplus}$ program (respectively, a $\mathbb{T}^{\mathrm{R}}$ program) $\vdash M:$ NAT $\rightarrow$ NAT which computes $f$ with at least probability $\frac{2}{3}$ of outputting the correct result $p \geq \frac{2}{3}$. Formally:

$$
\begin{array}{ccc}
f \in \mathbf{B P T}^{\oplus} \quad \text { iff } \quad \exists M \in \mathbb{T}^{\oplus}(\mathrm{NAT} \rightarrow \mathrm{NAT}), \quad \forall n \in \mathbb{N}, \quad \llbracket M \boldsymbol{n} \rrbracket(\boldsymbol{f}(\boldsymbol{n})) \geq \frac{2}{3} \\
f \in \mathbf{B P T}^{\mathrm{R}} \quad \text { iff } \quad \exists M \in \mathbb{T}^{\mathrm{R}}(\mathrm{NAT} \rightarrow \mathrm{NAT}), \quad \forall n \in \mathbb{N}, \quad \llbracket M \boldsymbol{n} \rrbracket(\boldsymbol{f}(\boldsymbol{n})) \geq \frac{2}{3}
\end{array}
$$

As is well known in complexity theory, the bound $\frac{2}{3}$ is arbitrary and we could have used equivalently any bound strictly above $\frac{1}{2}$. It is also natural to consider $\frac{1}{2}$ as a bound, but force the probability of error be strictly below it. We can then obtain the following classes of probabilistically representable functions:

$$
\begin{array}{cccc}
f \in \mathbf{P T}^{\oplus} & \text { iff } \quad \exists M \in \mathbb{T}^{\oplus}(\mathrm{NAT} \rightarrow \mathrm{NAT}), \quad \forall n \in \mathbb{N}, \quad \llbracket M n \rrbracket(f(n))>\frac{1}{2} \\
f \in \mathbf{P T}^{\mathrm{R}} \quad \text { iff } \quad \exists M \in \mathbb{T}^{\mathrm{R}}(\mathrm{NAT} \rightarrow \mathrm{NAT}), \quad \forall n \in \mathbb{N}, \quad \llbracket M n \rrbracket(f(\boldsymbol{n}))>\frac{1}{2}
\end{array}
$$

The pertinence of these classes is however controversial. Indeed, it can well be that $(M \boldsymbol{n})$ evaluates into $\boldsymbol{f}(\boldsymbol{n})$ with probability at least $\frac{1}{2}+h(n)$ where $h$ is an uncomputable function.

Due to the functional aspect of the considered objects ${ }^{5}$, we can nonetheless consider subclasses of $\mathbf{P} \mathbf{T}^{\oplus}$ (rep. $\mathbf{P} \mathbf{T}^{\mathrm{R}}$ ) for a reasonable dynamic bound $h$, namely one which can itself be computed in $\mathbb{T}$. We obtain this way the following dynamic Monte Carlo classes

$$
\begin{gathered}
f \in \mathbf{B P T}_{\geq \mathbb{T}}^{\oplus} \quad \text { iff } \quad \exists h \in \mathbf{D T}, \quad \exists M \in \mathbb{T}^{\oplus}(\mathrm{NAT} \rightarrow \mathrm{NAT}), \quad \forall n \in \mathbb{N}, \quad \llbracket M \boldsymbol{n} \rrbracket(\boldsymbol{f}(\boldsymbol{n}))>\frac{1}{h(n)} \\
f \in \mathbf{B P T}_{\geq \mathbb{T}}^{\mathrm{R}} \quad \text { iff } \quad \exists h \in \mathbf{D T}, \exists M \in \mathbb{T}^{\mathrm{R}}(\mathrm{NAT} \rightarrow \mathrm{NAT}), \quad \forall n \in \mathbb{N}, \quad \llbracket M \boldsymbol{n} \rrbracket(\boldsymbol{f}(\boldsymbol{n}))>\frac{1}{h(n)}
\end{gathered}
$$

There are easy inclusions between the just introduced classes of functions, since probabilistic observations are more liberal than dynamic Monte Carlo, themselves more liberal than Monte Carlo:

$$
\mathbf{D T} \subseteq \mathbf{B P} \mathbf{T}^{\oplus} \subseteq \mathbf{B P} \mathbf{T}_{\geq \mathbb{T}}^{\oplus} \subseteq \mathbf{P} \mathbf{T}^{\oplus} \quad \mathbf{D T} \subseteq \mathbf{B P} \mathbf{T}^{\mathrm{R}} \subseteq \mathbf{B P} \mathbf{T}_{\geq \mathbb{T}}^{\mathrm{R}} \subseteq \mathbf{P} \mathbf{T}^{\mathrm{R}}
$$

Is any of the above inclusions strict? In presence of binary probabilistic choice, the answer is negative:

Theorem 5.1. $\mathrm{PT}^{\oplus}=\mathrm{BPT}^{\oplus}=\mathrm{BPT}_{\geq \mathbb{T}}^{\oplus}=\mathrm{DT}$.

Proof. Let $f \in \mathbf{P} \mathbf{T}^{\oplus}$. There is $M \in \mathbb{T}^{\oplus}(\mathrm{NAT} \rightarrow \mathrm{NAT})$ such that $\llbracket M \boldsymbol{m} \rrbracket(f(m))>\frac{1}{2}$. By Theorem 3.10, there are $F \in \mathbb{T}(\mathrm{NAT} \rightarrow \mathrm{NAT} \rightarrow \mathrm{BIN})$ and $G \in \mathbb{T}(\mathrm{NAT} \rightarrow \mathrm{NAT})$ such that

$$
\forall k \leq \mathbb{N F}(G n), \quad \mathbb{N F}(F \boldsymbol{n} \boldsymbol{k})>\frac{1}{2} \Leftrightarrow k=f(n) .
$$

In this case can we set $M^{\prime} \in \mathbb{T}(\mathrm{NAT} \rightarrow \mathrm{NAT})$ such that $\mathbb{F}\left(M^{\prime} \boldsymbol{n}\right)=f(n)$, by:

$$
M^{\prime}:=\lambda n \cdot \operatorname{rec}\left\langle F n \mathbf{0}, \lambda k y \cdot \text { ite }\left\langle\sup _{\frac{1}{2}}(F n k), k, y\right\rangle, G n\right\rangle
$$

\footnotetext{
$5_{\text {in }}$ contrast to what happened for the polynomial classes.
} 
where $\sup _{\frac{1}{2}}$ is testing whether the input is above $\frac{1}{2}$ :

$$
\begin{array}{rl}
\sup _{\frac{1}{2}}: \mathrm{NAT} \times \mathrm{NAT} \rightarrow \mathrm{NAT} & \sup _{\frac{1}{2}}:=\lambda\langle m, n\rangle .(m+m)>2^{n} \\
>_{-}: \mathrm{NAT} \times \mathrm{NAT} \rightarrow \mathrm{NAT} & M>N:=\operatorname{rec}\left\langle\lambda u . \mathbf{0}, \lambda x y u \cdot \operatorname{rec}\left\langle\mathbf{1}, \lambda a_{-} . y a, u\right\rangle, M\right\rangle N
\end{array}
$$

When considering countable probabilistic choice, we cannot quite get to the same result, but close to that:

Theorem 5.2. $\mathrm{BPT}_{\geq \mathbb{T}}^{\mathrm{R}}=\mathrm{BPT}^{\mathrm{R}}=\mathrm{DT}$.

Proof. Let $f \in \mathbf{B P T}_{\geq \mathbb{T}}^{\mathrm{R}}$. There is $M \in \mathbb{T}^{\mathrm{R}}(\mathrm{NAT} \rightarrow \mathrm{NAT})$ and $H \in \mathbb{T}(\mathrm{NAT} \rightarrow \mathrm{NAT})$ such that

$$
\llbracket M \boldsymbol{m} \rrbracket(f(m)) \geq \frac{1}{2}+\frac{1}{\mathrm{NF}(H \boldsymbol{m})} .
$$

By Theorem 4.8, there exists $F: \mathrm{NAT} \rightarrow \mathrm{NAT} \rightarrow \mathrm{NAT} \rightarrow \mathrm{BIN}$ and $Q: \mathrm{NAT} \rightarrow \mathrm{NAT} \rightarrow \mathrm{NAT}$ in $\mathbb{T}$ such that:

$$
\sum_{k \in \mathbb{N}}|\llbracket M \boldsymbol{m} \rrbracket(\boldsymbol{k})-\mathbb{N}(F \boldsymbol{m} \boldsymbol{n} \boldsymbol{k})| \leq \frac{1}{n} \quad \forall k \geq \mathbb{N F}(Q \boldsymbol{m} \boldsymbol{n}), \mathbb{N F}(F \boldsymbol{m} \boldsymbol{n} \boldsymbol{k})=\mathbf{0} .
$$

In particular, $f(m)$ is the only $k \leq \mathbb{F}(Q \boldsymbol{m}(H \boldsymbol{m}))$ such that:

$$
\mathrm{NF}(F \boldsymbol{m}(H \boldsymbol{m}) \boldsymbol{k})>\frac{1}{2}
$$

In this case can we define $N$ such that $\mathbb{N F}(N \boldsymbol{n})=f(n)$ by:

$$
N:=\lambda m \cdot \operatorname{rec}\left\langle F m(H m) \mathbf{0}, \lambda k y \cdot \operatorname{ite}\left\langle\sup _{\frac{1}{2}}(F m(H m) k), k, y\right\rangle, G m(H m)\right\rangle
$$

5.2. Las Vegas Observations. In Las Vegas observations, one requires the underlying program to either return a special value (by convention, we take $\mathbf{0}$ here) representing failure, or to return the correct value of the function, the latter with at least a certain probability of success. Mimicking what we have done for Monte Carlo observations in the previous section, we can thus define six classes of functions as follows:

$$
\begin{aligned}
& f \in \mathbf{L V} \mathbf{T}^{\oplus} \quad \text { iff } \quad \exists M \in \mathbb{T}^{\oplus}(\mathrm{NAT} \rightarrow \mathrm{NAT}) . \forall n \in \mathbb{N} . \llbracket M \boldsymbol{n} \rrbracket=\left\{\begin{array}{ccc}
\mathrm{S} \boldsymbol{f}(\boldsymbol{n}) & \mapsto & p \\
\mathbf{0} & \mapsto & (1-p)
\end{array}\right\} \text { with } p \geq \frac{1}{3} \\
& f \in \mathbf{L V T}^{\mathrm{R}} \quad \text { iff } \quad \exists M \in \mathbb{T}^{\mathrm{R}}(\mathrm{NAT} \rightarrow \mathrm{NAT}) . \forall n \in \mathbb{N} . \llbracket M \boldsymbol{n} \rrbracket=\left\{\begin{array}{ccc}
\mathrm{S} \boldsymbol{f}(\boldsymbol{n}) & \mapsto & p \\
\mathbf{0} & \mapsto & (1-p)
\end{array}\right\} \text { with } p \geq \frac{1}{3} \\
& f \in \mathbf{N T}^{\oplus} \quad \text { iff } \quad \exists M \in \mathbb{T}^{\oplus}(\mathrm{NAT} \rightarrow \mathrm{NAT}) . \forall n \in \mathbb{N} . \llbracket M \boldsymbol{n} \rrbracket=\left\{\begin{array}{ccc}
\mathrm{S} \boldsymbol{f}(\boldsymbol{n}) & \mapsto & p \\
\mathbf{0} & \mapsto & (1-p)
\end{array}\right\} \text { with } p>0 \\
& f \in \mathbf{N T}^{\mathrm{R}} \quad \text { iff } \quad \exists M \in \mathbb{T}^{\mathrm{R}}(\mathrm{NAT} \rightarrow \mathrm{NAT}) . \forall n \in \mathbb{N} . \llbracket M \boldsymbol{n} \rrbracket=\left\{\begin{array}{clc}
\mathrm{S} \boldsymbol{f}(\boldsymbol{n}) & \mapsto & p \\
\mathbf{0} & \mapsto & (1-p)
\end{array}\right\} \text { with } p>0 \\
& f \in \mathbf{L V} \mathbf{T}_{\geq \mathbb{T}}^{\oplus} \quad \text { iff } \quad \exists h \in \mathbf{D T} \exists M \in \mathbb{T}^{\oplus}(\mathrm{NAT} \rightarrow \mathrm{NAT}) . \forall n \in \mathbb{N} . \llbracket M \boldsymbol{n} \rrbracket=\left\{\begin{array}{ccc}
\mathrm{S} \boldsymbol{f}(\boldsymbol{n}) & \mapsto & p \\
\mathbf{0} & \mapsto & (1-p)
\end{array}\right\} \\
& \text { with } p \geq \frac{1}{h(n)}
\end{aligned}
$$




$$
\begin{gathered}
f \in \mathbf{L V} \mathbf{T}_{\geq \mathbb{T}}^{\mathrm{R}} \quad \text { iff } \quad \exists h \in \mathbf{D T} \exists M \in \mathbb{T}^{\mathrm{R}}(\mathrm{NAT} \rightarrow \mathrm{NAT}) . \forall n \in \mathbb{N} . \llbracket M \boldsymbol{n} \rrbracket=\left\{\begin{array}{ccc}
\mathrm{S} \boldsymbol{f}(\boldsymbol{n}) & \mapsto & p \\
\mathbf{0} & \mapsto & (1-p)
\end{array}\right\} \\
\text { with } p \geq \frac{1}{h(n)}
\end{gathered}
$$

The polynomial-time equivalent to, say, $\mathbf{L V} \mathbf{T}^{\oplus}$ or $\mathbf{L V} \mathbf{T}^{\mathrm{R}}$ are classes in the style of $\mathbf{Z P P}$, whose name comes from an equivalent presentation using zero-error probabilistic programs running in average-case polynomial time. Notice that the equivalence does not hold here. As usual the bound $\frac{1}{3}$ is arbitrary. The classes $\mathbf{N} \mathbf{T}^{\oplus}$ and $\mathbf{N} \mathbf{T}^{\mathrm{R}}$ in fact model a form of of nondeterministic observation: the actual value $\boldsymbol{f}(\boldsymbol{n})$ can be obtained with any probability, making the underlying notion of computation to collapse to may-convergence.

The same trivial inclusions between the introduced classes hold here:

$$
\mathbf{D T} \subseteq \mathbf{L V} \mathbf{T}^{\oplus} \subseteq \mathbf{L V} \mathbf{T}_{\geq \mathbb{T}}^{\oplus} \subseteq \mathbf{N} \mathbf{T}^{\oplus} ; \quad \mathbf{D T} \subseteq \mathbf{L V} \mathbf{T}^{\mathrm{R}} \subseteq \mathbf{L V} \mathbf{T}_{\geq \mathbb{T}}^{\mathrm{R}} \subseteq \mathbf{N} \mathbf{T}^{\mathrm{R}} .
$$

As for the reverse inclusions, a picture very similar to the one we had in Monte Carlo observations can be given here:

Theorem 5.3. $\mathbf{N T}^{\oplus}=\mathbf{L V T}^{\oplus}=\mathbf{L V T} \mathbf{T}_{\geq \mathbb{T}}^{\oplus}=\mathbf{D T}$.

Proof. Let $f \in \mathbf{N T}^{\oplus}$. There is $M \in \mathbb{T}^{\oplus}(\mathrm{NAT} \rightarrow \mathrm{NAT})$ such that $f(m)$ is the only $k \in \mathbb{N}$ such that $\llbracket M \boldsymbol{m} \rrbracket(\mathrm{S} \boldsymbol{k})>0$. By Theorem 3.10, there is $F \in \mathbb{T}(\mathrm{NAT} \rightarrow \mathrm{NAT} \rightarrow \mathrm{BIN})$ and $G \in \mathbb{T}(\mathrm{NAT} \rightarrow \mathrm{NAT})$ such that

$$
\forall k \leq \mathbb{N}(G n), \quad \mathbb{N}(F \boldsymbol{n}(\mathrm{S} k))>0 \quad \Leftrightarrow \quad k=f(n) .
$$

In this case can we set $N \in \mathbb{T}(\mathrm{NAT} \rightarrow \mathrm{NAT})$ such that $\mathbb{F}(N \boldsymbol{n})=f(n)$, by:

$$
N:=\lambda n \cdot \operatorname{rec}\left\langle F n \mathbf{0}, \lambda k y \cdot \mathrm{ite}\left\langle\sup _{0}(F n(\mathrm{~S} k)), k, y\right\rangle, G n\right\rangle
$$

where $\sup _{0}$ is testing whether the input is above $\frac{1}{2}$ :

$$
\sup _{0}: \mathrm{NAT} \times \mathrm{NAT} \rightarrow \mathrm{NAT} \quad \sup _{0}:=\lambda\langle m, n\rangle . m>\mathbf{0}
$$

Theorem 5.4. $\mathbf{L V T}_{\geq \mathbb{T}}^{\mathrm{R}}=\mathbf{L V T}^{\mathrm{R}}=\mathrm{DT}$.

Proof. Let $f \in \mathbf{L V T}_{\geq \mathbb{T}}^{\mathrm{R}}$. There is $M \in \mathbb{T}^{\mathrm{R}}(\mathrm{NAT} \rightarrow \mathrm{NAT})$ and $H \in \mathbb{T}(\mathrm{NAT} \rightarrow \mathrm{NAT})$ such that

$$
\llbracket M \boldsymbol{m} \rrbracket(\mathrm{S} \boldsymbol{k})>\mathbf{0} \quad \Leftrightarrow \quad \llbracket M \boldsymbol{m} \rrbracket(\mathrm{S} \boldsymbol{k})>\frac{1}{\operatorname{NF}(H \boldsymbol{m})} \quad \Leftrightarrow \quad k=f(m)
$$

By Theorem 4.8, there exists $F: \mathrm{NAT} \rightarrow \mathrm{NAT} \rightarrow \mathrm{NAT} \rightarrow \mathrm{BIN}$ and $Q: \mathrm{NAT} \rightarrow \mathrm{NAT} \rightarrow \mathrm{NAT}$ in $\mathbb{T}$ such that:

$$
\sum_{k \in \mathbb{N}}|\llbracket M \boldsymbol{m} \rrbracket(\boldsymbol{k})-\mathbb{N}(F \boldsymbol{m} \boldsymbol{n} \boldsymbol{k})| \leq \frac{1}{n} \quad \forall k \geq \mathbb{N}(Q \boldsymbol{m} \boldsymbol{n}), \mathbb{N}(F \boldsymbol{m} \boldsymbol{n} \boldsymbol{k})=\mathbf{0} .
$$

In particular, for $n=2 * \mathbb{F}(H \boldsymbol{m})$, we get that $f(m)$ is the only $k \leq \mathbb{F}(Q \boldsymbol{m}(\mathbf{2} *(H \boldsymbol{m})))$ such that:

$$
\mathbb{N F}(F \boldsymbol{m}(2 *(H \boldsymbol{m}))(\mathrm{S} \boldsymbol{k}))>\frac{1}{2 * \mathbb{N F}(H \boldsymbol{m})}
$$

In this case can we set $N$ such that $\mathbb{F}(N \boldsymbol{n})=f(n)$ by:

$$
\begin{aligned}
N:=\lambda m \cdot \operatorname{rec}\langle & F m(\mathbf{2} *(H m)) \mathbf{1}, \\
\lambda k y \cdot \mathrm{ite}\left\langle\sup _{\frac{1}{2}}\left((H \boldsymbol{m}) *_{b}(F m(\mathbf{2} *(H m))(\mathrm{S} k))\right), k, y\right\rangle, & \\
& G m(\mathbf{2} *(H m))\rangle
\end{aligned}
$$


where

$$
\begin{aligned}
(\cdot) *(\cdot): \mathrm{NAT} \times \mathrm{NAT} \rightarrow \mathrm{NAT} ; & M * N:=\operatorname{rec}\left\langle\mathbf{0}, \lambda \_y \cdot M+y, N\right\rangle ; \\
(\cdot) \star_{b}(\cdot): \mathrm{NAT} \times \mathrm{BIN} \rightarrow \mathrm{BIN} ; & \left(M *_{b}\right):=\lambda\langle m, n\rangle \cdot\langle(M * m, n)\rangle .
\end{aligned}
$$

5.3. On Probabilistic and Nondeterministic Observations. In the last two sections, we have not been able to precisely delineate the status of $\mathbf{P} \mathbf{T}^{\mathrm{R}}$ and $\mathbf{N} \mathbf{T}^{\mathrm{R}}$. As we previously mentioned, the practical pertinence of these classes is questionable, in the sense that the result will be obtained after an unbounded number of tries and the proof that the algorithm is correct is given as an oracle.

In this section, we exploit this intuition, by proving that both of them contain functions which are recursive bot not definable in $\mathbb{T}$. More precisely, we show that $\mathbf{N T}^{\mathrm{R}}$, the nondeterministic class over $\mathbb{T}^{\mathrm{R}}$, exactly captures (total) recursive functions, while $\mathbf{P} \mathbf{T}^{\mathrm{R}}$ has a bit more complex structure and corresponds to a recursive choice over two $\mathbb{T}$-definable possible results. Before giving these two results, a remark is in order: contrary to the polynomial case where $\mathbf{N P} \subseteq \mathbf{P P}$, we have $\mathbf{P T}^{\mathrm{R}} \subseteq \mathbf{N T}^{\mathrm{R}}$ here. In fact, in the realm of decision problems, the two classes collapse to the one of recursive decision problems. The difference between them can only be observed when considering proper functions, which are neglected in probabilistic complexity theory. For any subset $X$ of $\mathbb{N}$, the class $\operatorname{Rec}^{X}$ stands for the class of recursive total function whose range is included in $X$.

Theorem 5.5. $\mathrm{NT}^{\mathrm{R}}=\operatorname{Rec}^{\mathbb{N}}$

Proof. - Let us first consider the inclusion $\mathbf{N T}^{\mathrm{R}} \subseteq \mathbf{R e c} \mathbf{N}^{\mathbb{N}}$. Let $f \in \mathbf{N T}^{\mathrm{R}}=\mathbf{N} \mathbf{T}^{\mathrm{X}}$; there is $M \in \mathbb{T}^{\mathrm{X}}(\mathrm{NAT} \rightarrow \mathrm{NAT})$ such that $f(m)$ is the only $k \in \mathbb{N}$ such that $\llbracket M \boldsymbol{m} \rrbracket(\mathrm{S} \boldsymbol{k})>0$. This means that there is a finite execution of $M$ converging to ( $\mathbf{k} \boldsymbol{k})$. Thus we only have to perform a simple Breadth-first search in the binary execution tree of $M$.

- Now, consider the inclusion $\mathbf{N T}^{\mathrm{R}} \supseteq \mathbf{R e c}^{\mathbb{N}}$. Let $f \in \mathbf{R} \mathbf{e c}^{\mathbb{N}}$; then $f$ is computed by a program $M:$ NAT $\rightarrow$ NAT that makes use of the operators of System $\mathbb{T}$ and of unguarded recursion $\mathrm{Y}:(A \rightarrow A) \rightarrow A$; since the execution of $\boldsymbol{M n}$ is finite, there exists an error-free execution of $M[\mathrm{Y}:=\lambda x . \mathrm{X}\langle x, \perp\rangle] \boldsymbol{n} \in \mathbb{T}_{\perp}^{\mathrm{X}}$ that gives the same result; using an encoding of the error monad, we can easily get a term $N \in \mathbb{T}^{\mathrm{X}}(\mathbb{N} \rightarrow \mathbb{N})$ such that $f(m)$ is the only $k \in \mathbb{N}$ such that $\llbracket N \boldsymbol{m} \rrbracket(\mathrm{S} \boldsymbol{k})>0$. We conclude by $\mathbf{N} \mathbf{T}^{\mathrm{R}}=\mathbf{N} \mathbf{T}^{\mathrm{X}}$

Theorem 5.6. $f \in \mathbf{P T}^{\mathrm{R}}$ iff there are $g_{1}, g_{2} \in \mathbf{D T}$ and $h \in \mathbf{R e c}^{\{1,2\}}$ such that $f(n)=g_{h(n)}(n)$.

Proof. Let DT $\circ \mathbf{R e c}^{\{1,2\}}$ be the class of all those functions $f$ such that $f(n)=g_{h(n)}(n)$, where $g_{1}, g_{2} \in \mathbf{D T}$ and $h \in \mathbf{R e c}^{\{1,2\}}$. We prove the equality between $\mathbf{P T}^{\mathbf{R}}$ and $\mathbf{D T} \circ \mathbf{R e c}{ }^{\{1,2\}}$ as follows:

- First of all, $\mathbf{P} \mathbf{T}^{\mathrm{R}} \subseteq \mathbf{D T} \circ \mathbf{R e c}^{\{1,2\}}$. Let $f \in \mathbf{P T}^{\mathrm{R}}$; there is $M \in \mathbb{T}^{\mathrm{R}}(\mathrm{NAT} \rightarrow \mathrm{NAT})$ such that $\llbracket M \boldsymbol{m} \rrbracket(f(m))>\frac{1}{2}$. By Theorem 4.8 , there exists $F: \mathrm{NAT} \rightarrow \mathrm{NAT} \rightarrow \mathrm{NAT} \rightarrow \mathrm{BIN}$ and $Q: N A T \rightarrow$ NAT $\rightarrow$ NAT in $\mathbb{T}$ such that:

$$
\sum_{k \in \mathbb{N}}|\llbracket M \boldsymbol{m} \rrbracket(\boldsymbol{k})-\mathbb{N}(F \boldsymbol{m} \boldsymbol{n} \boldsymbol{k})| \leq \frac{1}{n} ; \quad \forall k \geq \mathbb{F}(Q \boldsymbol{m} \boldsymbol{n}), \mathbb{N}(F \boldsymbol{m} \boldsymbol{n} \boldsymbol{k})=\mathbf{0} .
$$

Then for $n=8$, we get that $\mathbb{N F}(F \boldsymbol{m} \boldsymbol{n} \boldsymbol{k})>\frac{3}{8}$ for $k=f(m)$ and for at most one other value (since the total has to be bellow $\left.\frac{9}{8}\right)$, both bellow $\mathbb{N F}(Q \boldsymbol{m} \boldsymbol{n})$. We can thus construct two terms $N_{1}, N_{2}: \mathbb{N} \rightarrow \mathbb{N}$ in $\mathbb{T}$ such that 


\begin{tabular}{|c|c|c|c|c|c|c|}
\hline DT & $=$ & $\mathbf{B P T}^{\oplus}$ & $=$ & $\mathbf{B P} \mathbf{T}_{\geq \mathbb{T}}^{\oplus}$ & $=$ & $\mathbf{P} \mathbf{T}^{\oplus}$ \\
\hline DT & $=$ & $\mathbf{B P T}^{\mathrm{R}}$ & $=$ & $\mathbf{B P T}_{\geq \mathrm{T}}^{\mathrm{R}}$ & $\subset$ & $\mathbf{P} \mathbf{T}^{\mathrm{R}}$ \\
\hline DT & $=$ & $\mathbf{L V T}^{\oplus}$ & $=$ & $\mathbf{L V} \mathbf{T}_{\geq \mathbb{T}}^{\oplus}$ & $=$ & $\mathbf{N} \mathbf{T}^{\oplus}$ \\
\hline DT & $=$ & $\mathbf{L V T}^{\mathrm{R}}$ & $=$ & $\mathbf{L V} \mathbf{T}_{\geq \mathrm{T}}^{\mathrm{R}}$ & $\subset$ & $\mathbf{N} \mathbf{T}^{\mathrm{R}}$ \\
\hline
\end{tabular}

Figure 7. Subrecursive Classes - A Summary of the Obtained Results

- $N_{1} \boldsymbol{m}$ gives the smaller of those $k$ such that $\mathbb{N F}(F \boldsymbol{m} \boldsymbol{n} \boldsymbol{k})>\frac{3}{8}$

- and $\mathrm{N}_{2} \boldsymbol{m}$ the bigger.

A recursive procedure can then easily choose which one between the two failures is the correct one.

- Moreover, $\mathbf{P} \mathbf{T}^{\mathrm{R}} \supseteq \mathbf{D T} \circ \mathbf{R e c}{ }^{\{1,2\}}$. Let $g_{1}, g_{2} \in \mathbf{D T}$ and a recursive function $h: \mathbb{N} \rightarrow\{1,2\}$. Trivially, we can write $G: \mathrm{NAT} \rightarrow \mathrm{NAT} \rightarrow \mathrm{NAT}$ in $\mathbb{T} \subseteq \mathbb{T}^{\mathrm{R}}$ such that $\mathbb{N}(G \mathbf{1} \boldsymbol{n})=g_{1}(n)$ and $\mathbb{F}(G \mathbf{2 n})=g_{2}(n)$. As we have seen in Theorem 5.5, $h \in \mathbf{N T}^{\mathrm{R}}$ and thus there is $M \in \mathbb{T}^{\mathrm{R}}(\mathrm{NAT} \rightarrow \mathrm{NAT})$ such that $f(m)$ is the only $k \in \mathbb{N}$ such that $\llbracket M \boldsymbol{m} \rrbracket(\mathrm{S} \boldsymbol{k})>0$. We thus set:

$$
N:=\lambda n . \operatorname{ite}\langle M n, G(M n) n,(G \mathbf{1} n) \oplus(G \mathbf{2} n)\rangle
$$

A summary of the introduced subrecursive classes and the obtained results is in Figure 7

\section{Conclusions}

This paper is concerned with the impact of adding various forms of probabilistic choice operators to a higher-order subrecursive calculus in the style of Gödel's $\mathbb{T}$. The three probabilistic choice operators we analyze in this paper are equivalent if employed in the context of untyped or Turing-powerful $\lambda$-calculi [DLZ12]. As an example, $\mathrm{X}$ can be easily expressed by way of $\oplus$, thanks to fixpoints. Moreover, there is no hope to get termination in any of those settings. We give evidence that this is not the case in a subrecursive setting.

We claim that all we have said in this paper could have been spelled out in a probabilistic variation of Kleene's primitive recursive functions, e.g. [DLZG14]. Going higher-order makes our results, and in particular the termination results from Sections 2 and 3, significantly stronger. This is one of the reasons why we have proceeded this way. Classically, subrecursion refers to the study of relatively small classes of computable functions lying strictly below the partially recursive ones, and typically consisting of total functions. In this paper, we have initiated a study of the corresponding notion of subrecursive computability in presence of probabilistic choice operators, where computation itself becomes a stochastic process.

However, we barely scratched the tip of the iceberg, since the kinds of probabilistic choice operators we consider here are just examples of the possible ways one can turn a deterministic calculus like $\mathbb{T}$ into a probabilistic model of computation. The expressiveness of $\mathbb{T}^{\oplus, R, X}$ is sufficient to encode most reasonable probabilistic operators, but what can we say about their own expressive power? For example, what about a ternary operator in which either of the first two operators is chosen with a probability which depends on the value of the third operator? This ternary operator would have the type Ter $: A \rightarrow A \rightarrow(\mathrm{NAT} \rightarrow \mathrm{NAT}) \rightarrow A$, where the third argument $z: \mathrm{NAT} \rightarrow \mathrm{NAT}$ is seen as a probability $p \in[0,1]$ (whose $n^{\text {th }}$ binary component is given by $(z \boldsymbol{n})$ ). The expressivity of $\mathbb{T}^{\mathrm{R}}$ is sufficient to encode Ter := $\lambda x y z \cdot r e c x$ 


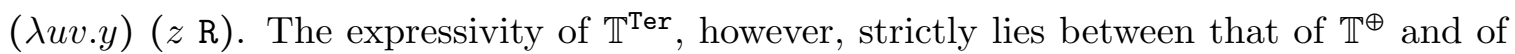
$\mathbb{T}^{\mathrm{R}}$ : $\mathbb{T}^{\mathrm{Ter}}$ can construct non binomial distributions ${ }^{6}$ while enforcing PAST. A general theory of probabilistic choice operators and of their expressive power is still lacking, and is an intriguing topic for future work.

Another research direction to which this paper hints at consists in studying the logical and proof-theoretical implications of endowing a calculus like $\mathbb{T}$ with probabilistic choice operators. The calculus $\mathbb{T}$ was born as a language of realizers for arithmetical formulas, and indeed the class of first-order functions $\mathbb{T}$ can express precisely corresponds to the ones which are provably total in Peano's arithmetic. But how about, e.g., $\mathbb{T}^{\mathrm{R}}$ ? Is there a way to characterize the functions (from natural numbers to distributions of natural numbers) which can be represented in it? Or even better: to which extent do real numbers in the codomain of a distribution in the form $\llbracket M \rrbracket$ (where $M$ is, say, a $\mathbb{T}^{\mathrm{R}}$ term of type NAT) are computable? They are of course computable in the sense of Turing computability, but how about subrecursive notions of real-number computability?

What is even more exciting, however, is the application of the ideas presented here to polynomial time computation. This would allow to go towards a characterization of expected polynomial time computation, thus greatly improving on the existing works on the implicit complexity of probabilistic systems [DLT15, DLZG14], which only deals with worst-case execution time. The authors are currently engaged in that.

Acknowledgements: We would like to thank Martin Avanzini and Charles Grellois for their precise comments and for their careful proofreading. The second author is partially supported by the ERC CoG DIAPASoN, GA 818616.

\section{REFERENCES}

[AB09] Sanjeev Arora and Boaz Barak. Computational Complexity: A Modern Approach. Cambridge University Press, 2009.

[Bar84] Henk P. Barendregt. The Lambda Calculus, Its Syntax and Semantics. SLFM, 1984.

[BG05] Olivier Bournez and Florent Garnier. Proving positive almost-sure termination. In RTA, volume 3467 of Lecture Notes in Computer Science, pages 323-337, 2005.

[CDL14] Raphä̈lle Crubillé and Ugo Dal Lago. On probabilistic applicative bisimulation and call-by-value $\lambda$-calculi. In ESOP, pages 209-228, 2014.

[DLFVY17] Ugo Dal Lago, Claudia Faggian, Benoît Valiron, and Akira Yoshimizu. The geometry of parallelism: classical, probabilistic, and quantum effects. In POPL 2017, pages 833-845. ACM, 2017.

[DLMSS56] Karel De Leeuw, Edward F Moore, Claude E Shannon, and Norman Shapiro. Computability by probabilistic machines. Automata studies, 34:183-198, 1956.

[DLT15] Ugo Dal Lago and Paolo Parisen Toldin. A higher-order characterization of probabilistic polynomial time. Inf. Comput., 241:114-141, 2015.

[DLZ12] Ugo Dal Lago and Margherita Zorzi. Probabilistic operational semantics for the lambda calculus. RAIRO - Theor. Inf. and Applic., 46(3):413-450, 2012.

[DLZG14] Ugo Dal Lago, Sara Zuppiroli, and Maurizio Gabbrielli. Probabilistic recursion theory and implicit computational complexity. Sci. Ann. Comp. Sci., 24(2):177-216, 2014.

[EPT14] Thomas Ehrhard, Michele Pagani, and Christine Tasson. Probabilistic Coherence Spaces are Fully Abstract for Probabilistic PCF. In P. Sewell, editor, POPL. ACM, 2014.

[FH15] Luis María Ferrer Fioriti and Holger Hermanns. Probabilistic termination: Soundness, completeness, and compositionality. In POPL, pages 489-501, 2015.

\footnotetext{
${ }^{6}$ Such as Ter $01(\operatorname{rec} \mathbf{0}(\lambda x \cdot \operatorname{rec} \mathbf{1}(\lambda y z \cdot \mathbf{0})))$.
} 
[Gil74] John T. Gill, III. Computational complexity of probabilistic turing machines. In Proceedings of STOC 1974, pages 91-95. ACM, 1974.

[GMR ${ }^{+}$08] Noah D. Goodman, Vikash K. Mansinghka, Daniel M. Roy, Keith Bonawitz, and Joshua B. Tenenbaum. Church: a language for generative models. In UAI, pages 220-229, 2008.

[GTL89] Jean-Yves Girard, Paul Taylor, and Yves Lafont. Proofs and Types. Cambridge University Press, 1989.

[HKSY17] Chris Heunen, Ohad Kammar, Sam Staton, and Hongseok Yang. A convenient category for higher-order probability theory. In LICS 2017, pages 1-12. IEEE Computer Society, 2017.

[JT98] Achim Jung and Regina Tix. The troublesome probabilistic powerdomain. Electr. Notes Theor. Comput. Sci., 13:70-91, 1998.

[KK15] Benjamin Lucien Kaminski and Joost-Pieter Katoen. On the hardness of almost-sure termination. In $M F C S$, volume 9234 of $L N C S$, pages 307-318, 2015.

[Koz81] Dexter Kozen. Semantics of probabilistic programs. J. Comput. Syst. Sci., 22(3):328-350, 1981.

[MM05] Annabelle McIver and Carroll Morgan. Abstraction, Refinement and Proof for Probabilistic Systems. Monographs in Computer Science. Springer, 2005.

[MR95] Rajeev Motwani and Prabhakar Raghavan. Randomized Algorithms. Cambridge University Press, 1995.

[MS99] Christopher D Manning and Hinrich Schütze. Foundations of statistical natural language processing, volume 999. MIT Press, 1999.

[Pap94] Christos H. Papadimitriou. Computational complexity. Addison-Wesley, 1994.

[Pea88] Judea Pearl. Probabilistic reasoning in intelligent systems: networks of plausible inference. Morgan Kaufmann, 1988.

[Pri12] Simon J. D. Prince. Computer Vision: Models, Learning, and Inference. Cambridge University Press, New York, NY, USA, 2012.

[San69] Eugene S Santos. Probabilistic Turing machines and computability. Proceedings of the American Mathematical Society, 22(3):704-710, 1969.

[SD78] N. Saheb-Djahromi. Probabilistic LCF. In MFCS, pages 442-451, 1978.

[Sta79] Richard Statman. The typed lambda-calculus is not elementary recursive. Theor. Comput. Sci., 9:73-81, 1979.

[SU06] Morten Heine Sørensen and Pawel Urzyczyn. Lectures on the Curry-Howard Isomorphism,. Elsevier Science Inc., New York, NY, USA, 2006. 